\title{
Heart failure causes cholinergic transdifferentiation of cardiac sympathetic nerves via gp130-signaling cytokines in rodents
}

\author{
Hideaki Kanazawa,1,2 Masaki leda,1,2 Kensuke Kimura, ${ }^{1}$ Takahide Arai,1,2 \\ Haruko Kawaguchi-Manabe,1 Tomohiro Matsuhashi,1,2 Jin Endo,,1,2 Motoaki Sano,1 \\ Takashi Kawakami,1,2 Tokuhiro Kimura, ${ }^{3}$ Toshiaki Monkawa, ${ }^{2}$ Matsuhiko Hayashi,4 \\ Akio Iwanami, ${ }^{5}$ Hideyuki Okano, ${ }^{5}$ Yasunori Okada, ${ }^{3}$ Hatsue Ishibashi-Ueda, ${ }^{6}$ \\ Satoshi Ogawa, ${ }^{2}$ and Keiichi Fukuda ${ }^{1}$
}

\begin{abstract}
'Department of Regenerative Medicine and Advanced Cardiac Therapeutics, 2Department of Internal Medicine, ${ }^{3}$ Department of Pathology, ${ }^{4}$ Department of Apheresis and Dialysis Center, and ${ }^{5}$ Department of Physiology, Keio University School of Medicine, Shinjuku-ku, Tokyo, Japan. ${ }^{6}$ Department of Pathology, National Cardiovascular Center, Osaka, Japan.
\end{abstract}

\begin{abstract}
Although several cytokines and neurotrophic factors induce sympathetic neurons to transdifferentiate into cholinergic neurons in vitro, the physiological and pathophysiological roles of this remain unknown. During congestive heart failure (CHF), sympathetic neural tone is upregulated, but there is a paradoxical reduction in norepinephrine synthesis and reuptake in the cardiac sympathetic nervous system (SNS). Here we examined whether cholinergic transdifferentiation can occur in the cardiac SNS in rodent models of CHF and investigated the underlying molecular mechanism(s) using genetically modified mice. We used Dahl salt-sensitive rats to model CHF and found that, upon CHF induction, the cardiac SNS clearly acquired cholinergic characteristics. Of the various cholinergic differentiation factors, leukemia inhibitory factor (LIF) and cardiotrophin-1 were strongly upregulated in the ventricles of rats with CHF. Further, LIF and cardiotrophin-1 secreted from cultured failing rat cardiomyocytes induced cholinergic transdifferentiation in cultured sympathetic neurons, and this process was reversed by siRNAs targeting Lif and cardiotrophin-1. Consistent with the data in rats, heart-specific overexpression of LIF in mice caused cholinergic transdifferentiation in the cardiac SNS. Further, SNS-specific targeting of the gene encoding the gp130 subunit of the receptor for LIF and cardiotrophin-1 in mice prevented CHF-induced cholinergic transdifferentiation. Cholinergic transdifferentiation was also observed in the cardiac SNS of autopsied patients with CHF. Thus, CHF causes target-dependent cholinergic transdifferentiation of the cardiac SNS via gp130-signaling cytokines secreted from the failing myocardium.
\end{abstract}

\section{Introduction}

Cardiac function is tightly controlled by the balance between the sympathetic nervous system (SNS) and the parasympathetic nervous system (PNS). The SNS produces norepinephrine (NE) and increases the heart rate, conduction velocity, and myocardial contraction and relaxation, while the PNS produces acetylcholine (Ach) that reduces cardiac performance. In congestive heart failure (CHF), sympathetic neural tone is upregulated, and excess SNS activation leads to pathophysiological effects, such as myocardial damage, decline of cardiac function, and lethal arrhythmia $(1,2)$, and also causes depletion of cardiac NE content (3). This depletion of NE in CHF has been considered to be the result of excess NE secretion, disturbance of NE reuptake, and loss of noradrenergic nerve terminals $(4,5)$. However, we recently reported that the attenuation of NE in CHF was caused by downregulation of NE synthesis, concomitant with the reduced NE reuptake (6). However, the molecular mechanisms underlying the reduction in catecholaminergic characteristics of cardiac SNS in CHF remain poorly understood.

Conflict of interest: The authors have declared that no conflict of interest exists. Citation for this article: J Clin Invest. 2010;120(2):408-421. doi:10.1172/JCI39778.
Neurotrophic factors that are secreted by the target organ and their target-derived retrograde signals are essential for the development and differentiation of neurons and well as for neuronal diversification and acquisition of neuronal properties (7). Previous studies have established that leukemia inhibitory factor (LIF), a member of the IL- 6 cytokine family, can induce cultured sympathetic neurons to switch neurotransmitters, from NE to Ach (8). Fukada purified a cholinergic differentiation factor from cultured rat heart cells that can induce neurotransmitter switching from NE to Ach in cultured sympathetic neurons $(9,10)$, and Yamamori confirmed that this factor was identical to LIF (11). LIF can induce neurotransmitter switching in vitro and in vivo. Although sweat glands are innervated by catecholaminergic sympathetic nerves at birth, the switch of neurotransmitter from catecholamine to Ach occurs gradually during development (7). Sympathetic neuron-specific gene targeting of gp130, an IL-6 cytokine family receptor, revealed that sympathetic nerves do not undergo cholinergic differentiation in the sweat gland (12). Studies in transgenic mice overexpressing LIF in pancreas show that the catecholaminergic characteristics decline, while the cholinergic characteristics increase (13). These results demonstrate that LIF/gp130 signaling plays an essential role in cholinergic neurotransmitter switching in sympathetic nerves. 
A Sympathetic nerve TH Actinin
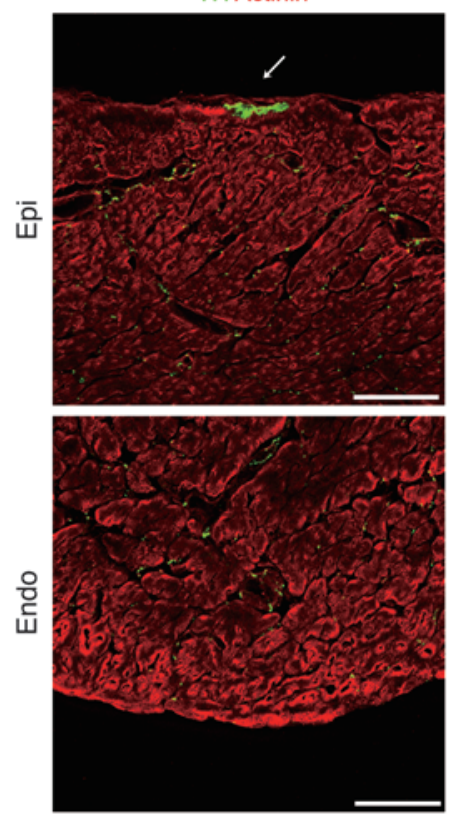

B

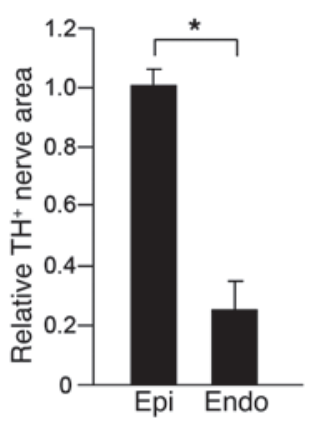

C

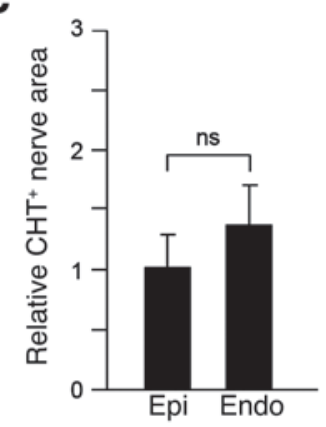

D
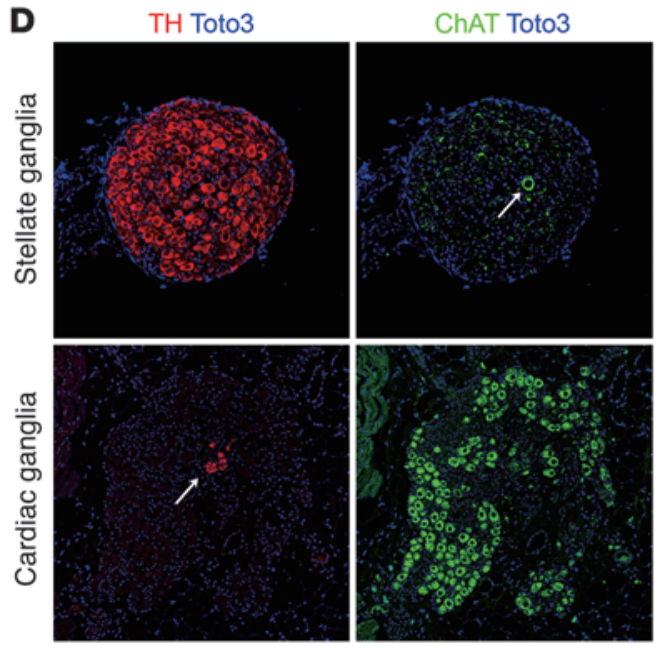

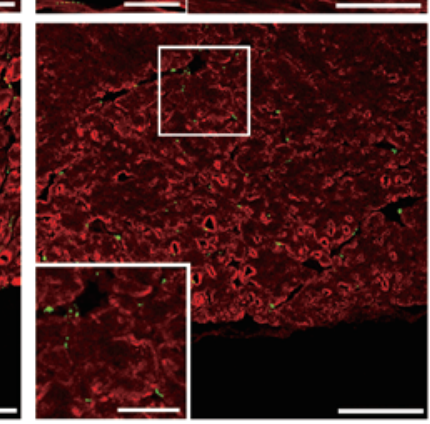
CHT Actinin
s.t.
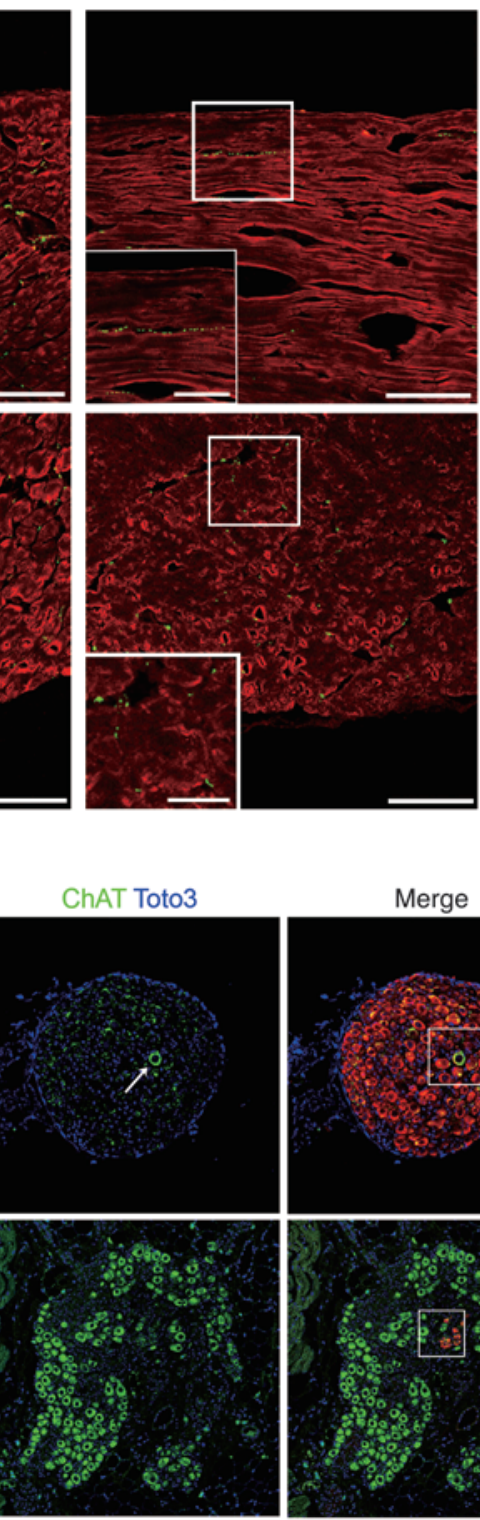

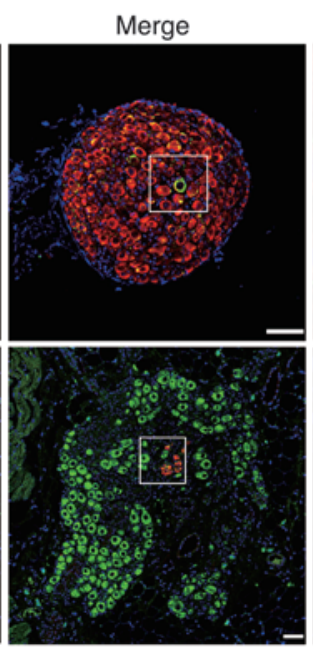

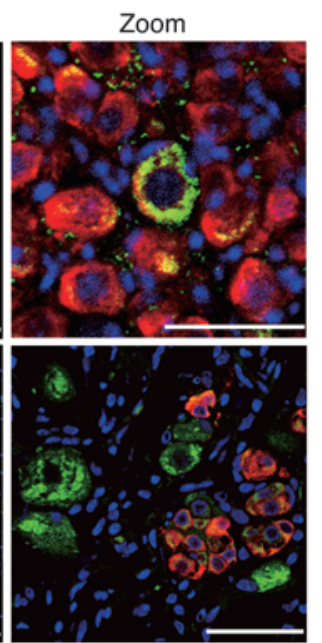

\section{Figure 1}

Distribution of sympathetic and parasympathetic nerves in the rat heart. (A) Immunofluorescence staining for $\mathrm{TH}, \mathrm{CHT}$ (green), and $\alpha$-actinin (red) in the rat LV. $\mathrm{TH}^{+}$ nerves were more abundant in the subepicardial layer (epi) than the subendocardial layer (endo). The arrow indicates sympathetic nerves at the epicardial surface. $\mathrm{No} \mathrm{CHT}^{+}$nerves were observed at the epicardial surface, and $\mathrm{CHT}^{+}$ nerves were more abundant in the subendocardial layer. Highermagnification views of the boxed regions are shown in the insets. (B) Quantitative analysis of $\mathrm{TH}^{+}$nerve area of LVs $(n=5)$. (C) Quantitative analysis of $\mathrm{CHT}^{+}$nerve area of LVs $(n=5)$. (D) Representative immunostaining for TH (red), ChAT (green), and Toto3 (blue) in rat stellate ganglia and cardiac ganglia. $A$ $\mathrm{ChAT}^{+}$neuron (arrow, top row) was surrounded by $\mathrm{TH}^{+}$neurons in the stellate ganglia. A few $\mathrm{TH}^{+}$cells (arrow, bottom row) were observed in cardiac ganglia. Higher-magnification views of the boxed regions in the third column are shown in the fourth column. Representative data are shown in each panel. ${ }^{*} P<0.01$. Scale bars: $100 \mu \mathrm{m}$ (A); $50 \mu \mathrm{m}$ (A, insets, and D).
CHF leads to upregulation of a range of growth factors and cytokines in the heart (14). LIF and other members of the IL- 6 family, which can induce fetal gene expression (so-called rejuvenation) in adult cardiomyocytes, are upregulated during $\mathrm{CHF}$ $(15,16)$. In cardiac SNS in CHF, we also observed strong expression of growth-associated protein 43 (GAP43) and highly polysialylated neural cell adhesion molecule (PSA-NCAM), which are immature neuronal markers expressed specifically in the fetal SNS. This result suggests that cardiac SNS dysfunction is accompanied by neuronal rejuvenation (6). To further understand the precise pathophysiological molecular mechanism, we investigated the relationship between phenotype and paracrine factors in failing myocardium.

In the present study, we show that cardiac SNS in CHF in human hearts and animal models, can cause neurotransmitter switching and transdifferentiation from catecholaminergic into cholinergic neurons, and this process is induced by gp130-mediated signaling via cholinergic differentiation factors released from failing myocardium.

\section{Results}

Distribution of sympathetic and parasympathetic nerves in the rat heart. We first defined the sympathetic and parasympathetic innervation of the rat heart. Immunostaining of LVs for tyrosine hydroxylase (TH) and choline transporter (CHT) was performed as a catecholaminergic and cholinergic nerve marker, respectively. TH-immunopositive $\left(\mathrm{TH}^{+}\right)$nerves were abundant in LVs, with greater innervation of the subepicardium (Figure 1, A and B) as we previously reported (17). $\mathrm{CHT}^{+}$nerves were fewer in number than $\mathrm{TH}^{+}$nerves, and their density was not different between myocardial layers (Figure 1, A and C). The thick and fasciculated nerve fibers at the epicardial surface expressed TH but not CHT (Figure 1A). 
A

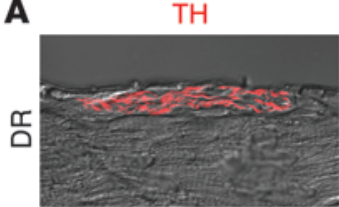

\&

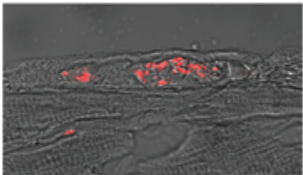

C

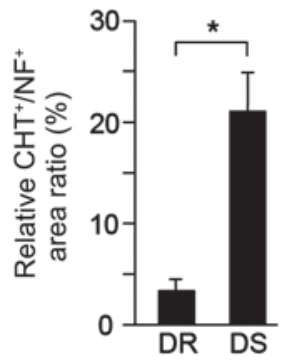

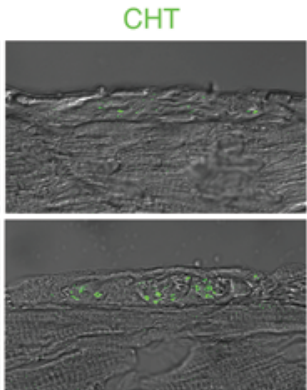

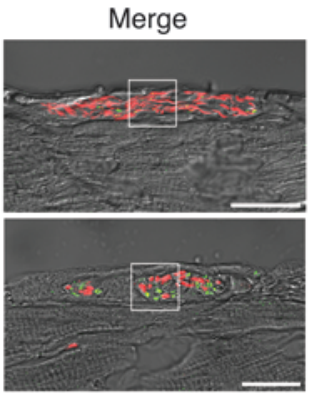

Zoom

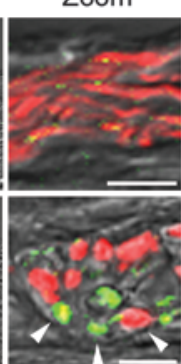

B

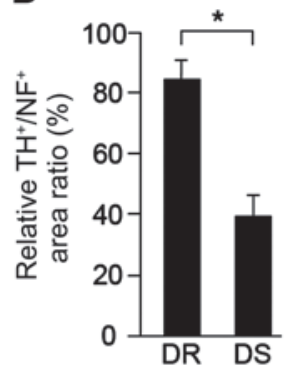

D

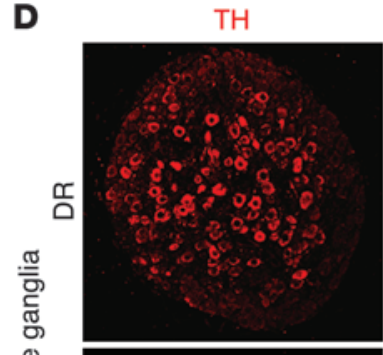

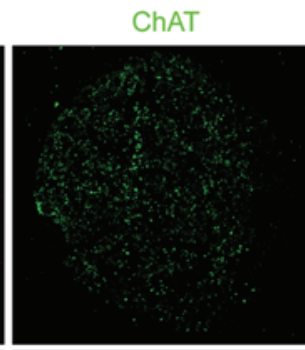

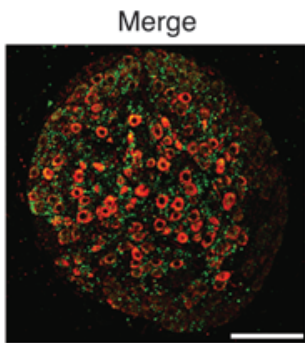

Zoom
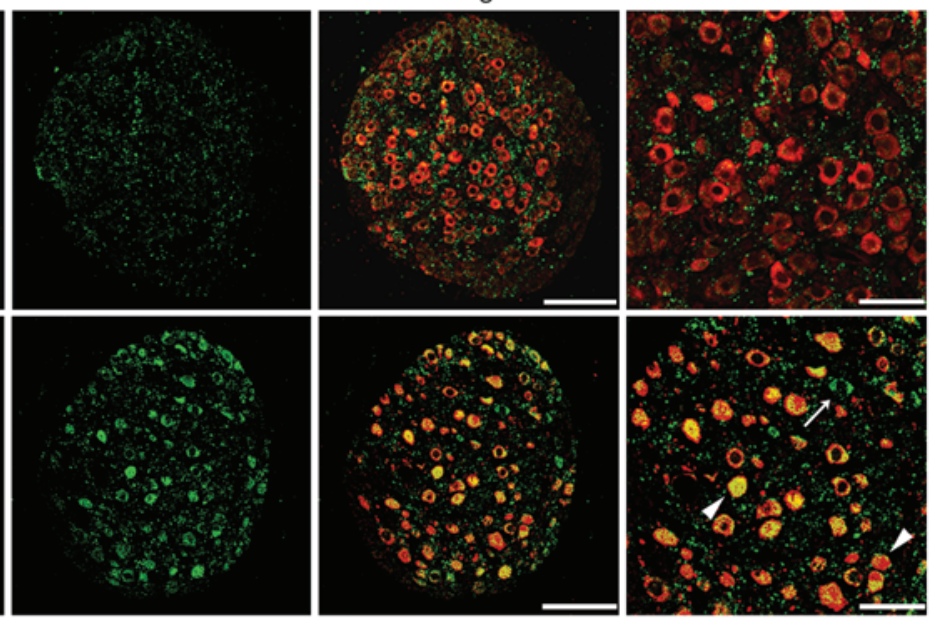

G

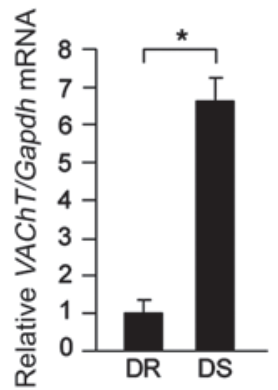

E

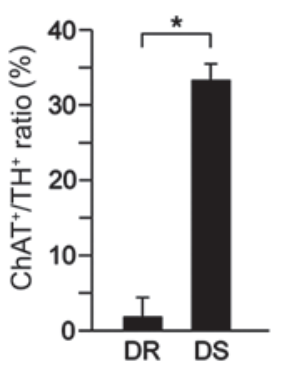

F

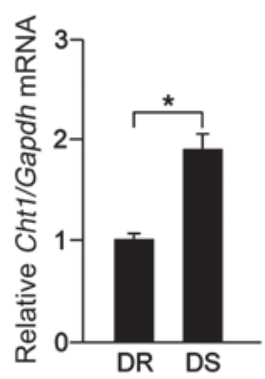

Figure 2

Cardiac nerve fibers and stellate ganglia neurons in DS rat ventricles have both catecholaminergic and cholinergic activity. (A) Confocal images of double immunofluorescence staining for TH (red) and $\mathrm{CHT}$ (green) in LVs at the epicardium in DR and DS rats. Numbers of $\mathrm{CHT}^{+}$nerve fibers (arrowheads) were increased, and some nerves coexpressed TH. Higher-magnification views of the boxed regions in the third column are shown in the fourth column. Representative data are shown in each panel. (B and C) Quantitative analysis of the $\mathrm{TH}^{+} / \mathrm{NF}^{+}$and $\mathrm{CHT}^{+} / \mathrm{NF}^{+}$area ratios in LVs at the epicardium $(n=5)$. (D) Double immunostaining with TH (red) and ChAT (green) of the stellate ganglia in DR and DS rats. Note that DS rats had more ChAT+ neurons. Some cells coexpressed TH (arrowheads), some did not (arrow). (E) Quantitative analysis of the $\mathrm{ChAT}^{+} / \mathrm{TH}^{+}$ ratio in the stellate ganglia $(n=4)$. (F) Cht1 expression in the stellate ganglia of DR and DS rats, determined by qRT-PCR $(n=4)$. (G) VAChT expression in the stellate ganglia of DR and DS rats, determined by qRT-PCR $(n=4)$. ${ }^{*} P<0.01$. Scale bars: $20 \mu \mathrm{m}(\mathbf{A}) ; 100 \mu \mathrm{m}$ (D); $5 \mu \mathrm{m}$ (A, zoom); $50 \mu \mathrm{m}$ (D, zoom).

In cardiac SNS, preganglionic and postganglionic synapses occur mainly in the stellate ganglia and partially in the upper thoracic ganglia. The vast majority of neurons in the stellate ganglia were $\mathrm{TH}^{+}$, and a few coexpressed choline acetyltransferase (ChAT, a cholinergic nerve marker). In contrast, almost all neurons in the cardiac ganglia were $\mathrm{ChAT}^{+}$, with a few expressing TH (Figure 1D). Thus, catecholaminergic sympathetic innervation is predominant in LVs, and the cardiac SNS and PNS extend from the neurons in stellate and cardiac ganglia, respectively.
Cardiac sympathetic nerve fibers show an increase in cholinergic markerpositive neurons in Dabl salt-sensitive rat ventricles. In Dahl salt-sensitive (DS) rats, high salt intake causes hypertension, cardiac hypertrophy, fibrosis, and DS rats are a common model of CHF. To determine whether cardiac innervation is altered in CHF, we analyzed sympathetic neuronal function in DS rats and the control Dahl salt-resistant (DR) rats (control). DS rats showed elevated blood pressure and heart rate as well as pleural effusion (Supplemental Table 1; supplemental material available online with this article; 
A
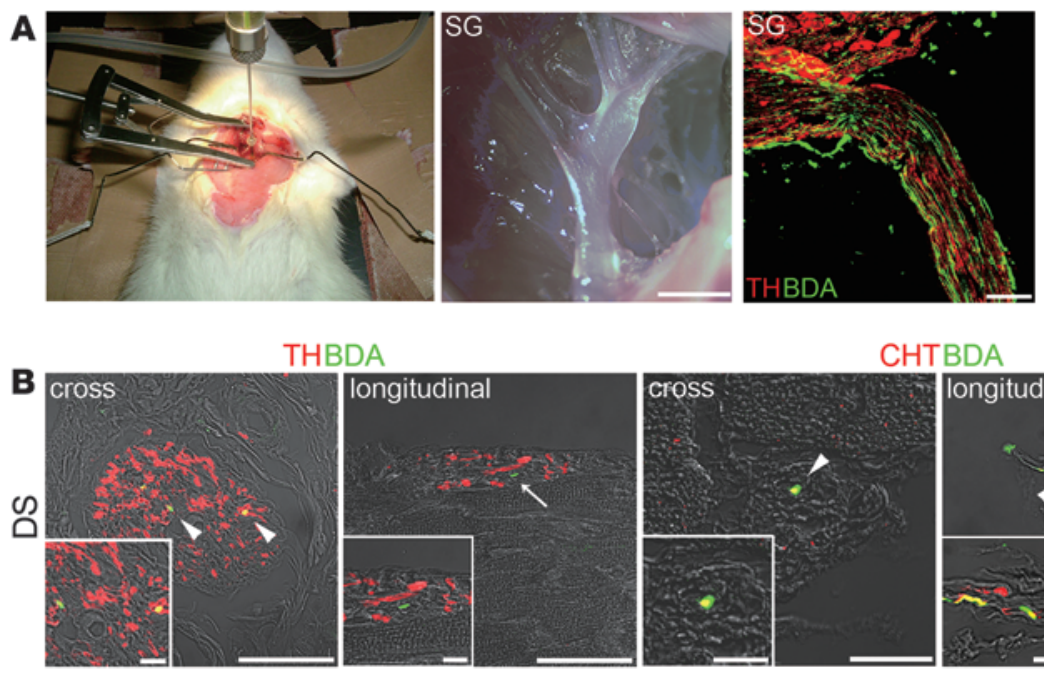

BDA
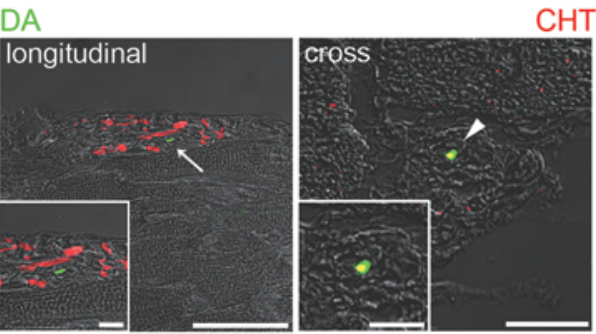

HTBDA

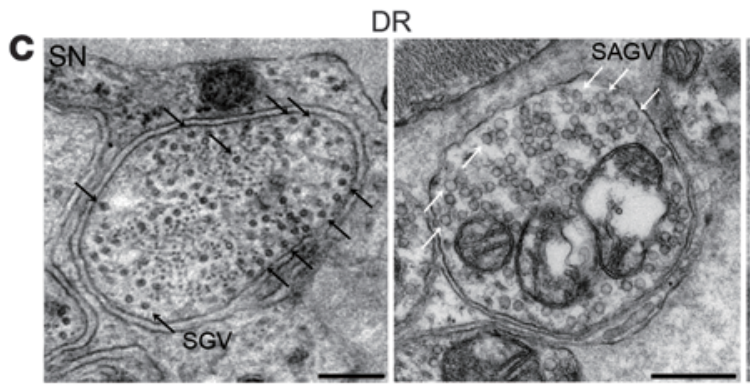

DR
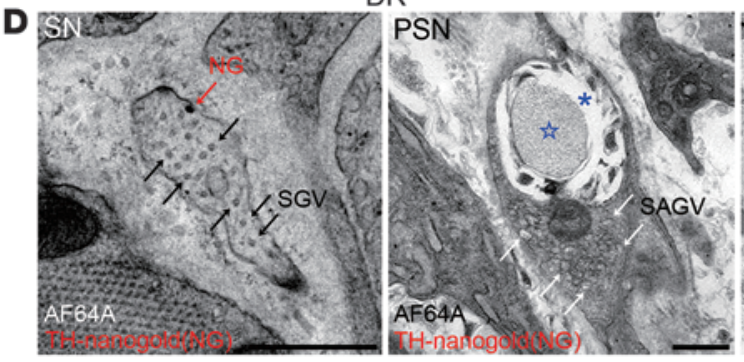

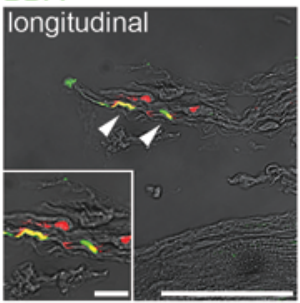

DS

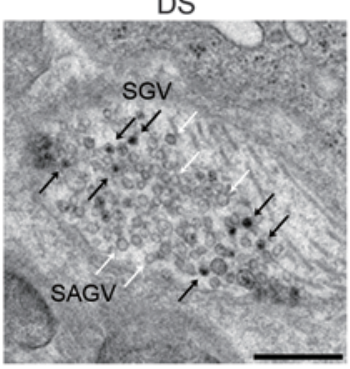

DS

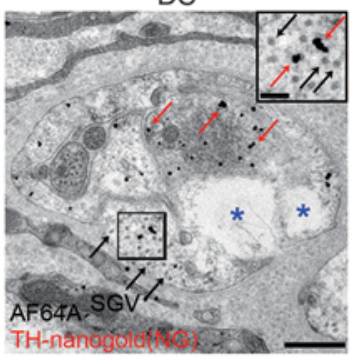

Figure 3

Cardiac sympathetic nerve fibers acquire cholinergic activity in the ventricles of rats with CHF. (A) BDA injection into the left stellate ganglion (left panel); a stereomicroscopic image of the stellate ganglion (middle panel); and BDA after injection, visualized as green signals (right panel) are shown. SG, stellate ganglia. (B) Epicardial sympathetic nerves in the DS rat LV. BDA (green, arrowheads) was transported to the cardiac sympathetic nerve fascicules. Some fibers coexpressed TH (red, arrowheads), but others did not (arrow). BDA+ nerve fibers (green, arrowheads) coexpressed $\mathrm{CHT}$, indicating $\mathrm{CHT}^{+}$ nerve fibers were derived from sympathetic nerves. Both cross and longitudinal sections are shown. (C) Transmission electron micrographs of the nerve endings in DR and DS rat LVs. Black arrows indicate SGVs in sympathetic nerve endings (SN). White arrows indicate SAGVs in parasympathetic nerve endings (PSN). (D) Immunoelectron micrographs of nerve endings in DR and DS rat LVs. Rats were injected with AF64A (cholinergic nerve fiber toxin), and the sections were immunostained with TH and silver-enhanced nanogold (NG) particles (red arrows). AF64A induced vacuoles (blue asterisks) and degeneration (blue star). The black arrows indicate SGVs. A higher-magnification view of the boxed region is shown in the inset. Representative data are shown in each panel. Scale bars: $2 \mathrm{~mm}$ (A, middle panel); $100 \mu \mathrm{m}$ (A, right panel); $50 \mu \mathrm{m}$ (B, left cross section panel and both longitudinal sections); $20 \mu \mathrm{m}$ (B, right cross section panel); $0.5 \mu \mathrm{m}$ (C and D); $10 \mu \mathrm{m}$ (B, insets); $0.1 \mu \mathrm{m}$ (D, inset).

doi:10.1172/JCI39778DS1). DS rats demonstrated heart-to-body weight ratios that were 1.7 -fold higher than those for DR rats at 15 weeks (Supplemental Figure 1A) and also showed dilated LVs and reduced fractional shortening (Supplemental Table 2). DS rats also showed elevated brain natriuretic peptide (BNP) expression and plasma NE concentrations but significantly lower cardiac NE concentrations, consistent with $\mathrm{CHF}$ (Supplemental Figure 1, B-D).

Triple-immunostaining revealed that DS LVs had fewer catecholaminergic nerves $\left(\mathrm{TH}^{+}\right)$and more cholinergic nerves $\left(\mathrm{CHT}^{+}\right)$than DR LVs (Supplemental Figure 1E). The epicardial sympathetic nerve bundles in DS rats show a marked increase in $\mathrm{CHT}^{+}$nerves and a marked decrease in $\mathrm{TH}^{+}$ nerves compared with DR rats (Figure $2 \mathrm{~A}$ and Supplemental Figure 2, A and B). Coexpression of CHT was observed in some $\mathrm{TH}^{+}$nerves. In $\mathrm{DR}$ rats, the majority of these fibers express $\mathrm{TH}\left(\mathrm{TH}^{+} /\right.$neurofilament ${ }^{+}\left[\mathrm{TH}^{+} / \mathrm{NF}^{+}\right]$ratio, $84 \%$ ), and a small proportion coexpress $\mathrm{CHT}$ $\left(\mathrm{CHT}^{+} / \mathrm{NF}^{+}\right.$ratio, $\left.3 \%\right)$. In contrast, DS rats had markedly more $\mathrm{CHT}^{+}$ nerves $\left(\mathrm{CHT}^{+} / \mathrm{NF}^{+}\right.$ratio, $\left.21 \%\right)$ and fewer $\mathrm{TH}^{+}$nerves $\left(\mathrm{TH}^{+} / \mathrm{NF}^{+}\right.$ratio, $39 \%$ ) than DR rats (Figure 2, B and $\mathrm{C}$ ). Double immunostaining for calcitonin gene-related peptide (a sensory nerve marker) and NF revealed that the density of sensory nerves in the LV of DS rats was not significantly different from that observed in DR rats (Supplemental Figure 2, C and D). We also performed double immunostaining for NF and dopamine $\beta$-hydroxylase $(\mathrm{DBH})$, another marker for sympathetic nerves (Supplemental Figure $3 \mathrm{~A})$. We found fewer catecholaminergic $\left(\mathrm{DBH}^{+}\right)$nerves in both the LV and stellate ganglia in DS rats than in DR rats. This result is consistent with our findings from $\mathrm{TH}$ immunostaining. These changes occur gradually with the progression of cardiac hypertrophy to CHF (Supplemental Figure 4). Taken together, these results show that markers of cholinergic signaling are increased in DS rats, and some cardiac sympathetic nerve fibers contain both catecholaminergic and cholinergic properties in the LV of DS rats.

Stellate ganglia neurons in rats with heart failure have predominantly cholinergic activity. Next, we investigated the expression of specific markers in the stellate ganglia. DR rats showed many $\mathrm{TH}^{+}$and $\mathrm{DBH}^{+}$neurons, a few ChAT ${ }^{+}$neurons, and many ChAT ${ }^{+}$presyn- 

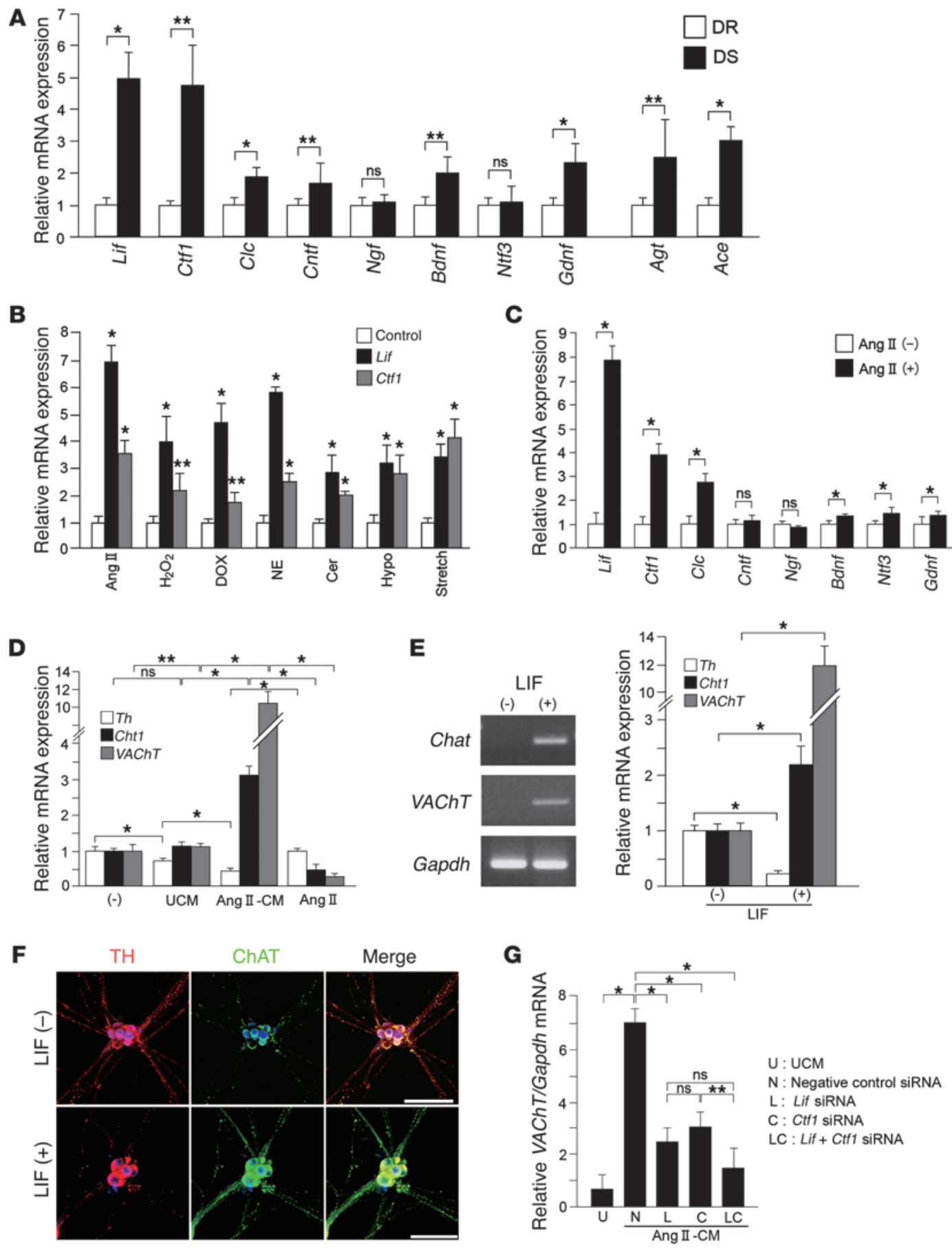

Figure 4

Cardiomyocytes secrete cholinergic differentiation factors that can induce neurotransmitter switching in cultured sympathetic neurons. (A) Expression of IL-6 family cytokines ( $\mathrm{Lif}, \mathrm{Ctf1}$, cardiotrophin-like cytokine [Clc], ciliary neurotrophic factor [Cntf]), neurotrophins (nerve growth factor [Ngf], brain-derived neurotrophic factor [Bdnf], neurotrophin-3 [Ntf3]), Gdnf, angiotensinogen (Agt), and angiotensin-converting enzyme (Ace) mRNA in DR and DS rat ventricles was determined by qRT-PCR $(n=4-6)$. (B) Primary cultured cardiomyocytes were stimulated with Ang II, $\mathrm{H}_{2} \mathrm{O}_{2}$, doxorubicin (DOX), NE, ceramide (Cer), hypoxia ( $5 \% \mathrm{O}_{2}$, Hypo), or $20 \%$ mechanical stretch. Expression of Lif and Ctf1 mRNA was investigated using qRT-PCR $(n=4)$. (C) Expression of IL-6 family cytokines and neurotrophins in Ang II-CMs were determined by qRT-PCR $(n=4)$. (D) Effect of unstimulated and Ang II-CM-conditioned media on the levels of expression of Th, Cht1, and VAChT in cultured sympathetic neurons $(n=3)$. (E) Effect of LIF on the levels of expression of Th, Cht1, and VAChT in cultured sympathetic neurons $(n=3)$. RT-PCR analysis of Chat and VAChT in cultured sympathetic neurons stimulated with LIF. (F) Immunocytochemical staining for TH, ChAT, and Toto3 in cultured sympathetic neurons. (G) Effect of preincubation of siRNA on the expression of Lif and Ctf1 on VAChT in cultured sympathetic neurons. Representative data are shown in each panel. ${ }^{*} P<0.01,{ }^{* \star} P<0.05$ compared with control $(\mathbf{B})$; ${ }^{*} P<0.01 ;{ }^{* \star} P<0.05(\mathbf{A}, \mathbf{C}-\mathbf{E}$, and $\mathbf{G})$. Scale bars: $100 \mu \mathrm{m}$. 
aptic nerve fibers. By comparison, DS rats had more abundant $\mathrm{ChAT}^{+}$neurons, some of which coexpressed $\mathrm{TH}$ and $\mathrm{DBH}$ (Figure 2, D and E, and Supplemental Figure 3B). CHT (Cht1) and vesicular Ach transporter (VAChT) expressions were also significantly higher (1.9-fold and 6.6-fold higher, respectively) in the stellate ganglia of DS rats (Figure 2, F and G). This shift toward cholinergic activity in DS rats was specific to the superior cervical, stellate, and upper thoracic ganglia. Lower thoracic and lumbar ganglia had no detectable ChAT expression (Supplemental Figure 5). Taken together, the cholinergic transdifferentiation of the cardiac SNS in CHF occurs not only at the peripheral axons but also the neuronal bodies themselves.

Functional evidence that the cardiac sympathetic nerves of DS rats switch from catecholaminergic to cholinergic activity. To confirm that the cardiac SNS undergoes a functional change from catecholaminergic to cholinergic activity, we labeled the cardiac SNS anterogradely from the stellate ganglia by injection of biotinylated dextran amine (BDA), an anterograde neuronal tracer. Both the sympathetic neurons in the stellate ganglia and the projecting nerve fibers were labeled with BDA (Figure 3A and Supplemental Figure 6A). In the $\mathrm{LV}$ of DR rats, all neurons injected with $\mathrm{BDA}^{+}$expressed $\mathrm{TH}$ (data not shown), whereas some other neurons either expressed $\mathrm{TH}$ or CHT in DS rats (Figure 3B).

Furthermore, direct observation of the nerve endings of the SNS by transmission electron microscopy showed that many small granular vesicles (SGVs, involving catecholamine) were observed in DR rats, consistent with previous studies (18), whereas the nerve endings in DS rats had both SGVs and small agranular vesicles (SAGVs, involving Ach) (Figure 3C and Supplemental Figure 6B). Quantification of the percentage of SGVs, found using the formula (SGV/[SGV + SAVG]), revealed that a substantial subset of cardiac SNS undergo partial transdifferentiation (Supplemental Figure 6C).

AF64A is a cholinergic nerve-specific toxin, which induces vacuolar degeneration of cholinergic nerve endings (19). We injected AF64A into the rats and analyzed the sympathetic nerve endings with immunoelectron microscopy, using anti-TH antibody. In DR rats, the catecholaminergic nerve endings, which were labeled with the TH immunogold particles, remained intact, and vacuolar degeneration was observed only at the cholinergic nerve endings, which were marked with SAGVs. In DS rats, vacuolar degeneration was also observed at the catecholaminergic nerve endings, which were labeled with TH immunogold particles and had both SGVs and SAGVs (Figure 3D and Supplemental Figure 6D). Thus, the SNS acquired functional cholinergic activity in CHF.

Cardiomyocytes can induce neurotransmitter switching in cultured sympathetic neurons by secretion of cholinergic differentiation factors. We then investigated whether neurotransmitter switching of the SNS in DS rats may be mediated by humoral factors, specifically IL-6 family cytokines, neurotrophins, and glial cellderived neurotrophic factor (GDNF), secreted from cardiomyocytes. Expression levels of Lif and cardiotrophin-1 (Ctf1) were significantly higher in the ventricles of DS rats than DR rats (approximately 5-fold higher), as was the expression of Gdnf (2-fold higher), but there was no difference in the levels of expression of neurotrophin-3 between animals with CHF and controls (Figure 4A).

We developed an in vitro model of heart failure by exposing cultured cardiomyocytes to various stimuli, including Ang II, $\mathrm{H}_{2} \mathrm{O}_{2}$, doxorubicin, NE, ceramide, hypoxia, and mechanical stretch. The effect of induced heart failure on Lif and Ctf1 mRNA was quantified.
All stimuli upregulated Lif and Ctf1, with the strongest effect observed for Ang II (Figure 4B). DS rat ventricles also showed upregulation of angiotensinogen $(A g t)$ and angiotensin-converting enzyme (Ace), indicating renin-angiotensin system is augmented with $\mathrm{CHF}$ (Figure 4A). Based on these findings, we treated the cultured cardiomyocytes with Ang II to mimic the failing myocardium. Ang II markedly augmented Lif and Ctf1 by 8.1- and 4.2-fold, respectively, but had a less significant effect on the other factors (Figure 4C).

Primary stellate ganglion neurons were then cultured in the presence of conditioned media from unstimulated cardiomyocytes (UCM) or Ang II-stimulated cardiomyocytes (Ang II-CMs). Ang IICMs markedly upregulated Cht1 and VAChT (3.2- and 11.5-fold increases, respectively) and markedly downregulated $T h$, while UCM had no effect (Figure 4D). Direct treatment of stellate ganglion neurons with Ang II did not induce any changes in cholinergic-related genes. Treatment of stellate ganglion neurons with LIF $(10 \mathrm{ng} / \mathrm{ml})$ had similar effects to those seen with conditioned media from stimulated cardiomyocytes; upregulation of Cht1, Chat, and VAChT and marked downregulation of $T h$ (Figure 4E). These results were confirmed by immunofluorescence staining (Figure 4F).

We then specifically inhibited Lif and/or Ctf1 expression by pretreatment of cardiomyocytes with siRNA before stimulation with Ang II. VAChT expression was reduced by $64 \%$ and $56 \%$ with siRNA specific for Lif and Ctf1, respectively, relative to their expression in cells treated with the negative control (scrambled) siRNA (Figure $4 \mathrm{G}$ ). Together, these results suggested that a combination of cholinergic differentiation factors secreted from hypertrophic cardiomyocytes, mainly LIF and CT-1, mediate neurotransmitter switching.

Cardiac-specific overexpression of LIF induces neurotransmitter switching in the cardiac SNS. We first generated LIF-loxP mice and crossed them with $\alpha$-myosin heavy chain-cardiac-specific Creexpressing ( $\alpha M H C$-Cre $)$ mice (Figure 5A). The LIF ${ }^{\alpha M H C-C r e}$ doubletransgenic mice specifically overexpress LIF in the heart (Figure $5 \mathrm{~B})$. We generated 13 double-transgenic lines, and selected lines 3 and 10 as representative lines that expressed LIF at different levels (Figure 5C and Supplemental Table 3). At 4 weeks of age, line 3 and 10 mice showed moderate and severe cardiac hypertrophy, respectively, whereas the $\alpha M H C$-Cre or LIF-loxP littermates did not show any obvious cardiac changes when compared to age-matched WT littermates. Body weight in line 10 was significantly decreased compared with the other mice.

WT, $\alpha M H C$-Cre, and Lif-loxP mice had abundant $\mathrm{TH}^{+}$nerves in LVs but very few $\mathrm{CHT}^{+}$nerves. $L I F^{\alpha M H C-C r e}$ line 10 mice had fewer $\mathrm{TH}^{+}$ nerves $\left(78 \%\right.$ of WT) and significantly more $\mathrm{CHT}^{+}$nerves by 8 fold, but there were no changes in neurotransmitter phenotype in line 3 (Figure 5, D and E). Cardiac NE concentration was also reduced in LIF ${ }^{\alpha M H C-C r e}$ hearts (line 10) (Figure 5F). Consistent with these results, the stellate ganglia in WT, $\alpha M H C-C r e$, and Lif-loxP mice only had a few $\mathrm{ChAT}^{+}$neurons, whereas LIFaMHC-Cre stellate ganglia (line 10) had abundant $\mathrm{ChAT}^{+}$neurons, some of which coexpressed TH (Figure $5, \mathrm{G}$ and $\mathrm{H}$ ). These show that cardiac-specific overexpression of LIF can induce neurotransmitter switching and cause cholinergic transdifferentiation in the cardiac SNS in vivo.

SNS-specific conditional gp130 gene-targeted mice prevent heart failure-induced cholinergic transdifferentiation. We investigated whether gp130-mediated signaling induces cholinergic transdifferentiation of SNS in CHF. SNS-specific EGFP reporter mice were generated by crossing $\mathrm{DBH}$-Cre recombinase transgenic ( $\mathrm{DBH}$-Cre) mice with EGFP-floxed mice, and expression of the EGFP in the adrenal gland, stellate ganglia and SNS was confirmed (Figure 6A). 
A

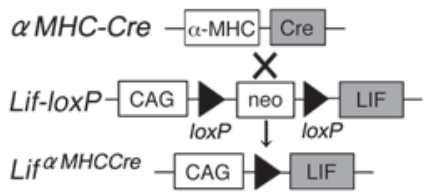

C

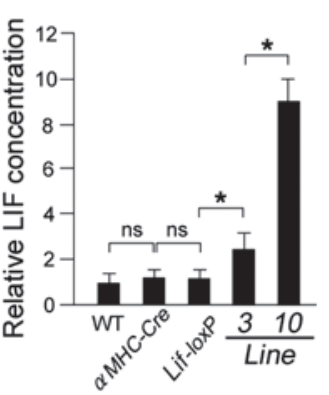

B

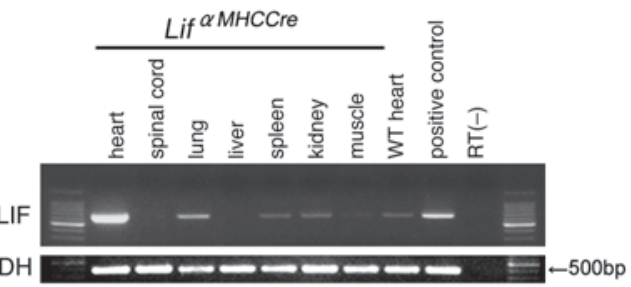

D

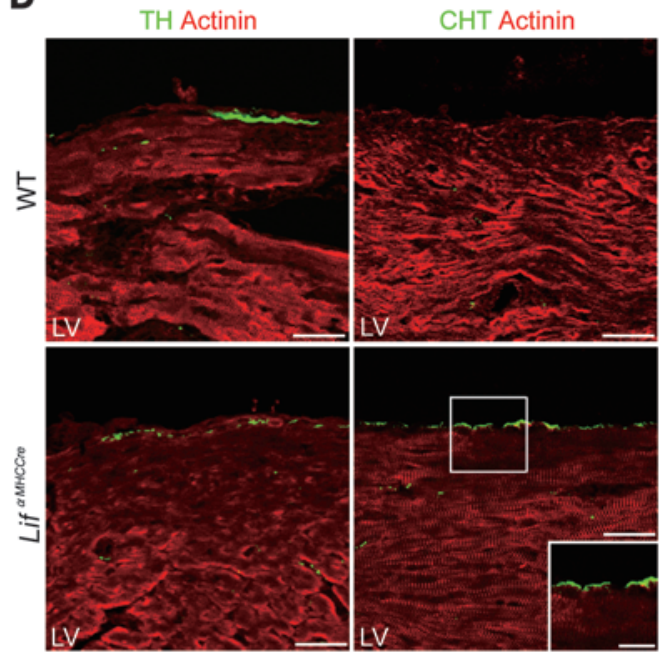

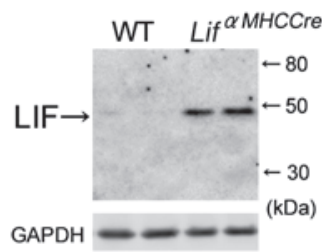

E

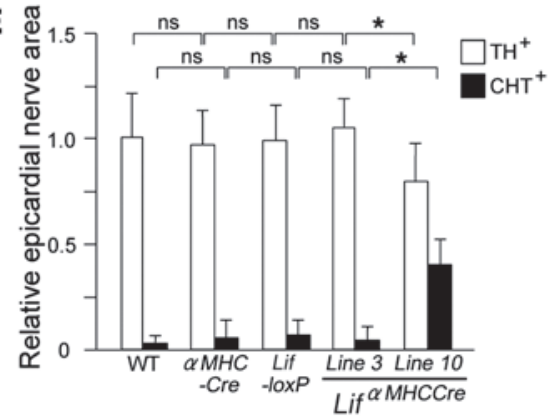

F

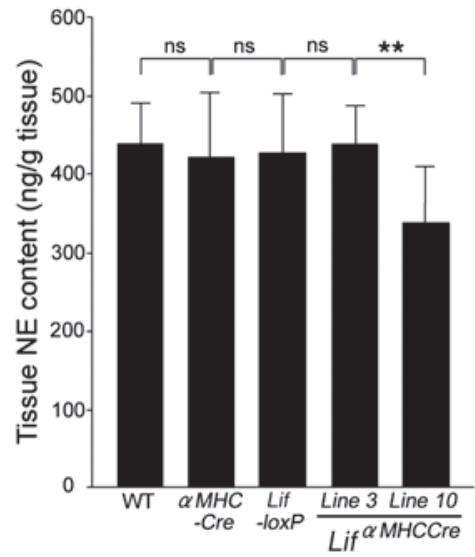

H

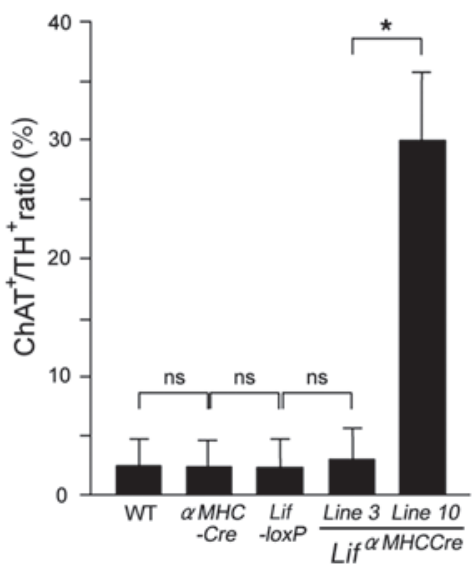

Figure 5

Cardiac-specific overexpression of LIF induces neurotransmitter switching in the cardiac sympathetic nervous system. (A) Schema of generation of LifaMHC-Cre mice using the Cre-loxP system. (B) RT-PCR and Western blot analysis of Lif mRNA expression in LifaMHC-Cre mice (line 10). (C) Quantitative analysis of LIF expression in lines 3 and 10. (D) Double immunofluorescence staining for $\alpha$-actinin (red), TH, or CHT (green) in WT and LifaMHC-Cre hearts (line 10). $\mathrm{TH}^{+}$nerves were decreased in LifaMHC-Cre hearts. In contrast, $\mathrm{CHT}^{+}$nerves were increased in LifaMHC-Cre hearts. A higher-magnification view of the boxed region is shown in the inset. (E) Quantitative analysis of $\mathrm{TH}^{+}$and $\mathrm{CHT}^{+}$nerve areas in the hearts of WT, $\alpha M H C$-Cre, and Lif-loxP mice and 2 lines of LifaMHC-Cre mice $(n=5)$. (F) NE content was lower in the ventricles of LifaMHC-Cre mice (line 10) than in those of WT, $\alpha M H C$-Cre, and Lif-loxP littermates or line 3 mice. $(n=5)$. (G) Representative immunostaining for TH (red), ChAT (green), and Toto3 (blue) in WT and LifaMHC-Cre stellate ganglia (line 10). (H) Quantitative analysis of ChAT+/ $\mathrm{TH}^{+}$ratios in the stellate ganglia in WT, $\alpha M H C$ Cre, Lif-loxP, and LifaMHC-Cre (lines 3 and 10) mice $(n=3)$. Representative data are shown in each panel. ${ }^{*} P<0.01 ;{ }^{* \star} P<0.05$. Scale bars: $50 \mu \mathrm{m}$ (D); $100 \mu \mathrm{m}$ (G); $25 \mu \mathrm{m}$ (D, inset). 
A

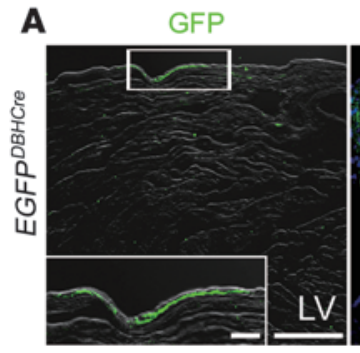

c
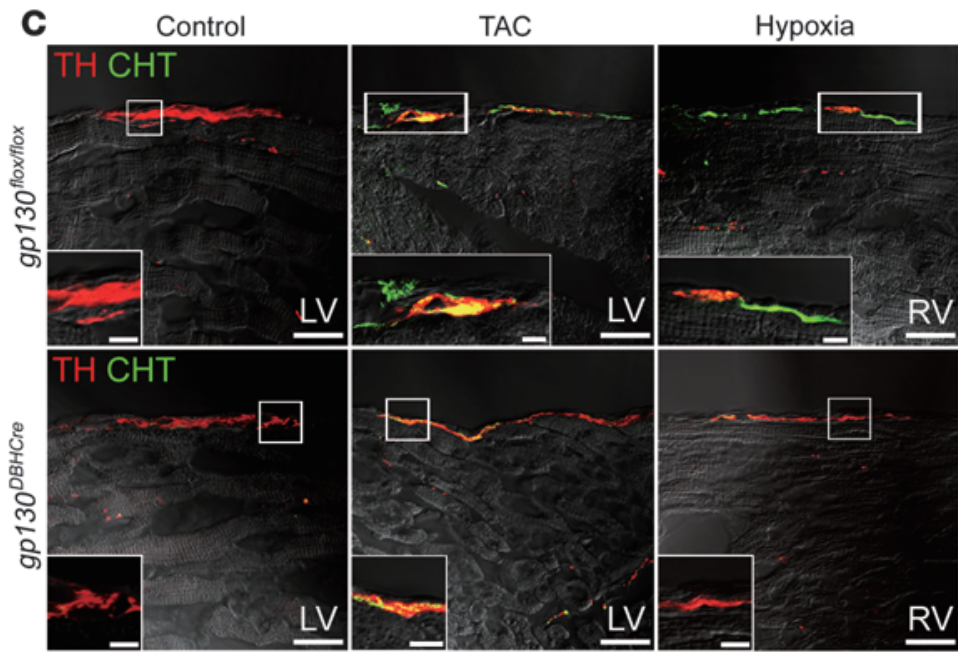

E

E
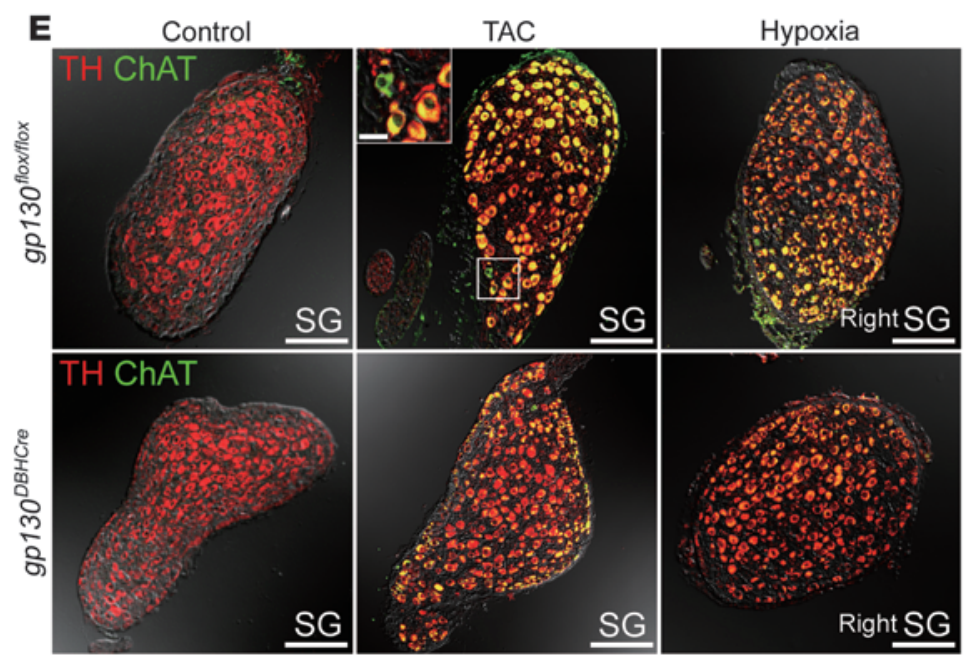

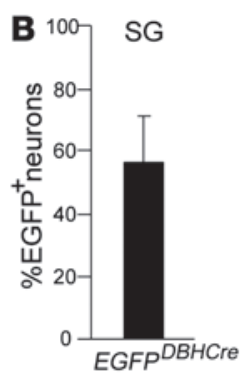

D
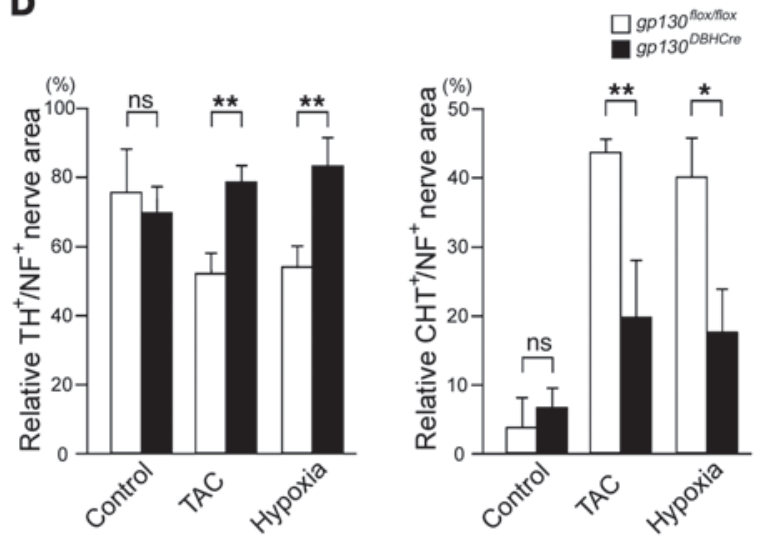

$\mathbf{F}$

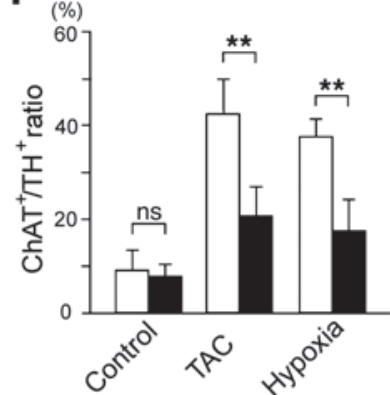

Figure 6

Conditional gp130 gene targeting in sympathetic nerves prevents heart failure-induced cholinergic transdifferentiation. (A) Immunofluorescent staining in the LV, stellate ganglia, and adrenal grand (AG) of EGFPDBH-Cre mice. Green and blue signals indicate GFP and Toto3 (nucleus), respectively. (B) Quantitative analysis of the percentage of EGFP+ neurons in EGFPDBH-Cre mice $(n=3)$. (C) Conditional gp130 gene-targeted mice (gp130 $\mathrm{DBH}$-Cre mice) and control mice (gp130flox/flox mice) were subjected to heart failure according to the TAC or hypoxia models. Immunofluorescent staining with anti-TH (red) or anti-CHT (green) antibody was performed on failing or control myocardium. (D) Quantitative analysis of the $\mathrm{TH}^{+} / \mathrm{NF}^{+}$and $\mathrm{CHT}^{+} / \mathrm{NF}^{+}$area ratios in $\mathrm{LVs}$ and $\mathrm{RVs}$ is indicated $(n=4)$. (E) gp130DBH-Cre and gp130flox/flox mice were subjected to heart failure, and immunofluorescent staining with TH (red) and ChAT (green) was performed on the stellate ganglia. (F) Quantitative analysis of the $\mathrm{ChAT}^{+} / \mathrm{TH}^{+}$ratio in stellate ganglia is shown $(n=4)$. Higher-magnification views of the boxed regions are shown in the insets $(\mathbf{A}, \mathbf{C}$, and $\mathbf{E})$. Scale bars: $100 \mu \mathrm{m}$ (A and $\mathbf{E}) ; 20 \mu \mathrm{m}$ (C and insets in $\mathbf{A}$ and $\mathbf{E}) ; 5 \mu \mathrm{m}\left(\mathbf{C}\right.$, insets). ${ }^{*} P<0.01 ;{ }^{* *} P<0.05$.

We demonstrated that $57 \%$ of the neuronal bodies of the stellate ganglia were marked with EGFP, thereby validating the efficiency of these transgenic mice (Figure 6B). SNS-specific gp130 gene-targeted mice were generated by crossing $\mathrm{DBH}$-Cre and gp130-floxed (gp130floxfflox) mice. The resultant $g p 130^{D B H-C r e}$ mice developed normally, and, under normal physiological conditions, the stellate ganglia and cardiac SNS stained with TH but not CHT, a finding consistent with control gp $130^{f l o x}$ flox mice (Figure 6, C and E). 
A

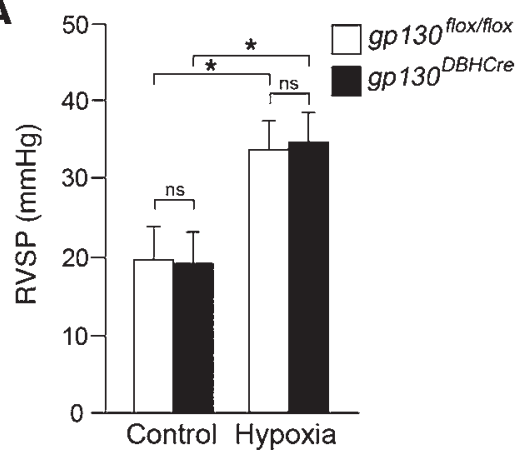

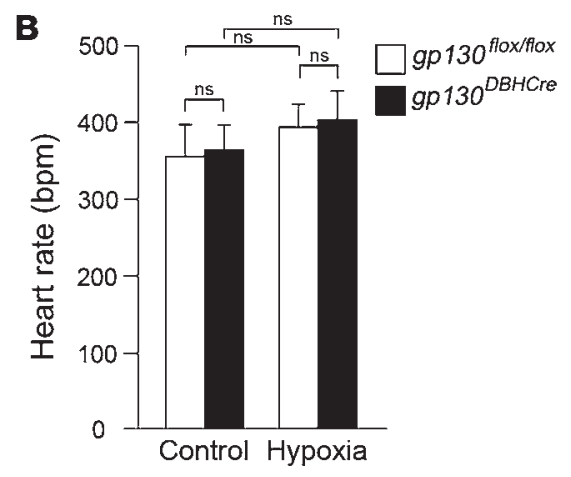

C
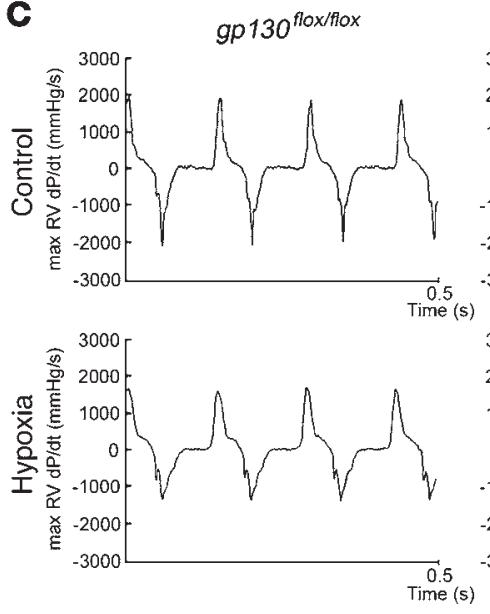
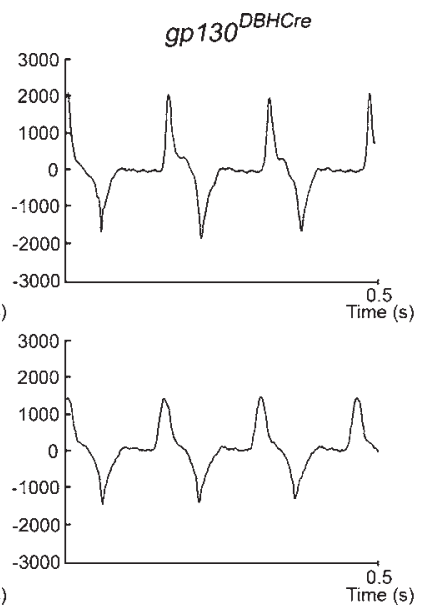

D

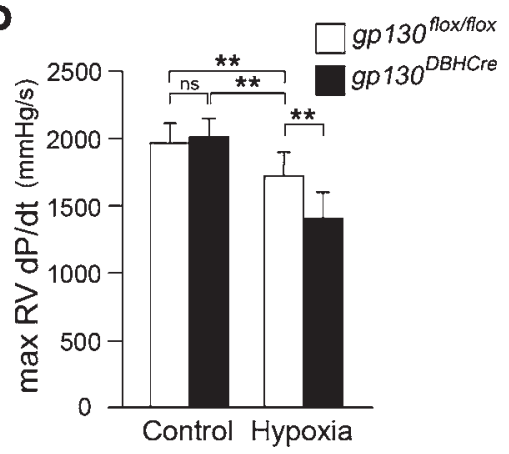

E

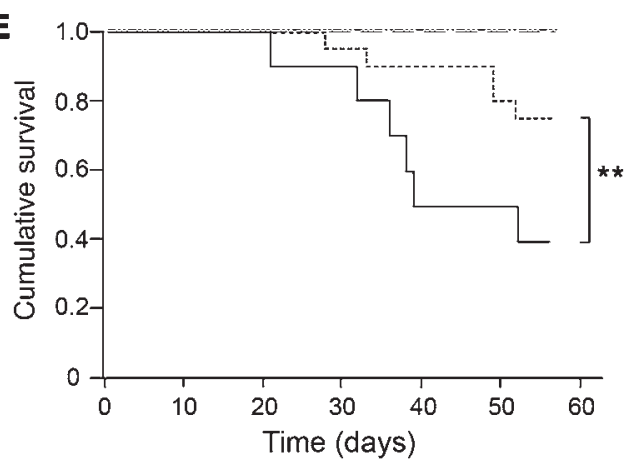

$\left.\begin{array}{l}\text { - gp } 130^{\text {fox/fox }} \\ - \text { gp } 130^{\text {DBHCre }}\end{array}\right\}$ Control

-..... go $130^{\text {flox/7ox }}$

gp $\left.130^{\text {hioxinox }}\right\}$ Hypoxia

\section{Figure 7}

Cardiac function and Kaplan-Meier analysis of gp130floxfllox and gp130 DBH-Cre mice under chronic hypoxia. (A) Measurement of the RVSP under chronic hypoxia at 8 weeks. (B) Measurement of the heart rate under chronic hypoxia at 8 weeks. (C) Representative recordings of maximum (max) $\mathrm{RVdP} / \mathrm{dt}$ under chronic hypoxia at 8 weeks. (D) Quantitative analysis of maximum RVdP/dt under control conditions and under chronic hypoxia at 8 weeks. (E) Kaplan-Meier analysis of the cumulative survival under control conditions and under chronic hypoxia. ${ }^{*} P<0.01 ;{ }^{* *} P<0.05$.

Chronic transverse aortic constriction and sustained hypoxia were used to generate a model of pressure overload-induced LV and RV heart failure, respectively (Supplemental Figure 7, A-F). Pressure gradient caused by transaortic constriction (TAC) was shown in Supplemental Figure 7, G and H. The baseline cardiac parameters of $g p 130^{D B H-C r e}$ mice, including heart weight/body weight ratio, heart rate, blood pressure, RV systolic pressure (RVSP), maximum $\mathrm{LVdP} / \mathrm{dt}$, and maximum RVdP/dt, were not significantly different from those of $g p 130^{f t o x} / f b x$ mice (Supplemental Figure 8). gp130foxffox mice showed cholinergic transdifferen- tiation in cardiac SNS and stellate ganglia, consistent with the rat $\mathrm{CHF}$ model. These changes were rescued in $g p 130^{\mathrm{DBH}-\mathrm{Cre}}$ mice (Figure 6, C and E). Quantitative analysis revealed that the relative $\mathrm{TH}^{+} / \mathrm{NF}^{+}$nerve area of the ventricular SNS in $g p 130^{D B H}$-Cre mice returned to the control level, while the relative $\mathrm{CHT}^{+} / \mathrm{NF}^{+}$nerve area was significantly reduced (Figure $6 \mathrm{D}$ ). The $\mathrm{ChAT}^{+} / \mathrm{TH}^{+}$ratio of the stellate ganglia was significantly reduced in $g p 130^{D B H}$-Cre mice (Figure 6F). We demonstrated that $57 \%$ of the neuronal bodies of the stellate ganglia were marked with EGFP, thereby validating the efficiency (Cre expression) of these transgenic mice. These 

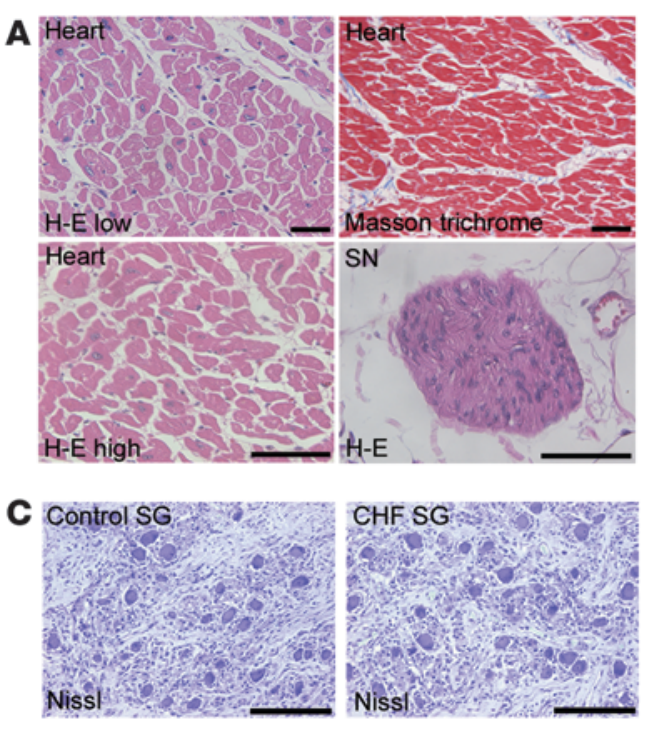

B
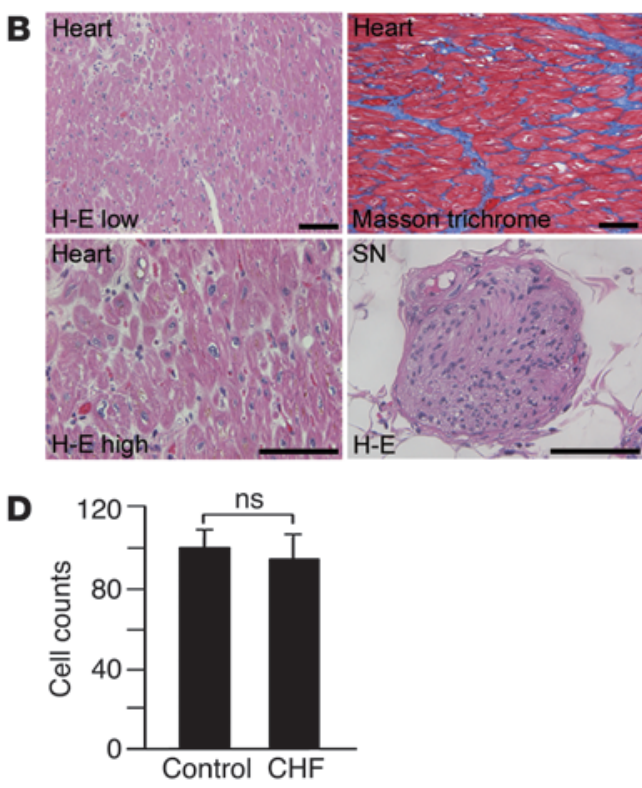

\section{Figure 8}

Histological analysis of human sample. (A) H\&E and Masson trichrome staining of the LV and cardiac sympathetic nerves in the control group. SN, sympathetic nerve; H-E low, H\&E staining with low magnification; $\mathrm{H}-\mathrm{E}$ high, $\mathrm{H} \& \mathrm{E}$ staining with high magnification. (B) H\&E and Masson trichrome staining of the LV and cardiac sympathetic nerves in the heart failure group. (C) Nissl staining of stellate ganglia in the control and CHF patients. (D) Quantitative analysis of stellate ganglia neuron numbers in the control and heart failure group $(n=5)$. Representative data are shown in each panel. Scale bars: $100 \mu \mathrm{m}$ (A and B); $200 \mu \mathrm{m}$ (C). results suggest that cholinergic transdifferentiation of the SNS in $\mathrm{CHF}$ is regulated primarily by gp130-signaling pathway.

We also evaluated cardiac function in $g p 130^{\text {flox/flox }}$ and $g p 130^{D B H-C r e}$ mice to determine whether cholinergic transdifferentiation has a significant effect in the setting of chronic hypoxia-induced heart failure. In chronic hypoxia, RVSP was significantly elevated in both $g p 130^{\text {flox/flox }}$ and $g p 130^{D B H-C r e}$ mice to levels that were not significantly different from each other (Figure 7A). Furthermore, there was no significant difference in heart rate between $g p 130^{\text {flox } / \text { flox }}$ and $g p 130^{D B H-C r e}$ mice (Figure $7 \mathrm{~B}$ ). The maximum $\mathrm{RVdP} / \mathrm{dt}$ was significantly lower in both $g p 130^{D B H-C r e}$ and $g p 130^{\text {flox/flox }}$ mice than in controls, which is indicative of RV heart failure. Interestingly, the maximum $\mathrm{RVdP} / \mathrm{dt}$ was significantly lower in $g p 130^{D B H-C r e}$ mice than in gp130 flox/flox mice (Figure 7, C and D). Moreover, KaplanMeier analysis revealed a significant difference in the cumulative survival between $g p 130^{f l o x} / f l o x$ and $g p 130^{D B H-C r e}$ mice subjected to chronic hypoxia. These results suggested that cholinergic transdifferentiation of the cardiac SNS via gp130 signaling might play a pivotal role in improving cardiac function during the development and progression of heart failure.

Neurotransmitter switching of the buman cardiac SNS in CHF. To determine whether our observations held true in humans, we examined the neurotransmitter phenotype of the cardiac SNS in patients with CHF (Figure 8 and Table 1). Consistent with the experimental results, the epicardial nerve bundles of control hearts had a predominance of $\mathrm{TH}^{+}$nerves, whereas $\mathrm{CHT}^{+}$nerves were predominant in $\mathrm{CHF}$ hearts, with some nerves coexpressing both markers. The same neurotransmitter phenotypes were seen in the stellate ganglia (Figure 9), confirming that the CHF patients undergo a switch from predominant catecholaminergic to cholinergic activity.

\section{Discussion}

The inherent plasticity of sympathetic neurons is widely accepted $(8-10,18,20,21)$. The neurotransmitter plasticity is also characteristic of other neuron types, such as dorsal root ganglia sensory neurons (22). However, here we demonstrate that neu- rotransmitter switching or cholinergic transdifferentiation of the cardiac SNS can also occur in a pathophysiological event, $\mathrm{CHF}$, in the adult. These results suggest cardiac SNSs have dual potential and the capacity to switch neurotransmitter, according to environmental conditions. Furthermore, we have identified the mechanism for this process; failing cardiomyocytes secrete a host of factors that can drive the SNS toward cholinergic activity by gp130-mediated signaling pathway.

This cholinergic transdifferentiation in cardiac SNS can explain some long-standing questions about CHF. The failing heart requires sympathetic stimulation to increase cardiac performance but, paradoxically, shows depletion of NE (3). This was initially thought to be due to excess NE secretion $(23,24)$, but recent studies have suggested that it arises from decreased NE reuptake $(25,26)$ and decreased $\mathrm{NE}$ synthesis $(6) . \mathrm{TH}^{+}$nerves are reduced in hearts after CHF, leading to the assumption that the SNS is anatomically denervated (5, 27-29). Conversely, Liang reported that there was a discrepancy between anatomical integrity and the catecholaminergic properties of the cardiac SNS in CHF, and that nerve fibers expressing the general neuronal marker, protein gene product 9.5, are intact, despite an overall reduction in catecholaminergic neurotransmitters (30). We have previously shown that in the cardiac SNS in CHF, the expression of $\mathrm{TH}$ and $\mathrm{DBH}$ is downregulated, with concomitant upregulation of GAP43 or PSA-NCAM proteins (6). The cholinergic transdifferentiation can explain these various observations. We propose that the neurotransmitter switching to the cholinergic phenotype might contribute to NE depletion and can explain the discrepancy between the anatomical profile and catecholaminergic properties of the cardiac SNS in CHF. It remains unknown why cholinergic transdifferentiation occurs. One possibility is that it is a side effect. Since LIF and CT-1 have a cardioprotective effect, their upregulation may be required for the survival of a failing heart, and this upregulation may accidentally cause transdifferentiation. Another possibility is that cholinergic transdifferentiation of the SNS in CHF is an adaptive response to protect the heart from excessive sympathetic discharge, similar to the way that 


\section{Table 1}

Human sample characteristics

\begin{tabular}{lcccc} 
Case no. & Cause of death & Age (years) & Sex & Heart weight (g) \\
Control & & & & \\
1 & AML & 45 & M & 440 \\
2 & MDS & 33 & M & 350 \\
3 & MDS & 39 & M & - \\
4 & ML & 58 & M & 350 \\
5 & Pneumonia & 78 & M & 320 \\
6 & Liver cancer & 45 & M & 300 \\
7 & Pancreatic cancer & 57 & M & 420 \\
8 & Esophageal cancer & 52 & M & 350 \\
Heart failure & & & & \\
1 & OMl & 81 & M & 468 \\
2 & DCM, HTP & 50 & M & - \\
3 & OMI & 74 & M & 655 \\
4 & DCM, HTP & 38 & F & - \\
5 & Myocarditis & 39 & M & 415 \\
6 & OMl & 76 & M & 545 \\
7 & DCM, HTP & 43 & F & - \\
8 & DCM & 62 & M & 660 \\
\hline
\end{tabular}

Patients in the control group had no evidence of heart disease. All patients in the heart failure group had $\mathrm{CHF}$. Heart weight was significantly higher in the heart failure group than the control group $(P<0.01)$. AML, acute myeloid leukemia; DCM, dilated cardiomyopathy; $F$, female; HTP, heart transplantation; M, male; ML, malignant lymphoma; MDS, myelodysplastic syndrome; OMI, old myocardial infarction.

critically involved in this process. We observed that CHF-induced cholinergic transdifferentiation is rescued by sympathetic neuron-specific gp130-targeted mice. This finding suggests that gp130-mediated cytokines, LIF and CT-1, play a pivotal role in cholinergic transdifferentiation of cardiac SNS.

This research began with the use of DS rats as a model for heart failure, because this model could produce heart failure with high reproducibility, without variation in the severity of heart failure, because this model can be obtained without surgical treatment. In addition, we could not perform the experiment with the neuronal tracer using the mouse model. However, gain of function and loss of function was necessary to clarify the molecular mechanisms underlying heart failure. Thus, we used the genetic mouse model for our experiments.

The clinical significance of cholinergic transdifferentiation in the cardiac SNS in CHF remains unclear, but further clarification of the mechanisms that mediate these phenomena may represent a novel step toward potential therapies in patients with CHF.

\section{Methods}

Animal experiments. Six-week-old DS and DR rats and six- to eight-week-old Wistar rats were purchased

$\beta$ receptor downregulation, $\beta$ adrenergic receptor kinase, and beta blockers are protective. Our findings showing the chronic hypoxiainduced RV failure in $g p 130^{D B H-C r e}$ mice suggest that cholinergic transdifferentiation of cardiac sympathetic nerves might be an adaptive response to protect the heart. Supporting this notion, cholinergic signaling via muscarinic Ach receptors has been shown to promote specific cytoprotective signaling pathways and to improve cardiac function $(31,32)$. Other studies have shown that augmentation of parasympathetic nerve activity decreased mortality rates with $\mathrm{CHF}$, thus suggesting a beneficial effect of cholinergic signaling in CHF (33). Taken together, these findings support our idea that cholinergic transdifferentiation is an adaptive response. Further analysis is required to draw firm conclusions on the importance of cholinergic transdifferentiation in modulating cardiac function.

LIF was first identified as a cholinergic differentiation factor from the conditioned medium of cardiomyocytes (11) and also has a range of specific roles in a variety of cell types $(34,35)$. Recent reports have shown that other members of the IL- 6 cytokine family (12), neurotophin-3 (36), and GDNF (37) can induce cholinergic differentiation. The present study found that a combination of cholinergic differentiation factors, particularly LIF and CT-1, are critical for this neurotransmitter change in cardiac SNS. LIF has previously been shown to be upregulated in cardiac hypertrophy and $\mathrm{CHF}(38,39)$. Our study also determined the level of expression of LIF required for induction of neurotransmitter switching. Comparison of LIF-transgenic mice at different levels suggested there may be a threshold concentration required to induce transdifferentiation. The expression level of LIF in DS rats was less than 8.9 fold (line 10), but we still observed the acquisition of cholinergic characteristics, suggesting that additional factors may be from Japan CLEA. Rats were fed a high-salt diet $(8 \% \mathrm{NaCl})$ for 9 weeks. All experimental procedures and protocols were approved by the Animal Care and Use Committees of Keio University.

Generation of transgenic mice expressing LIF in the heart. LIF cDNA was subcloned into pCALNL5 plasmid (a gift from I. Saito, University of Tokyo, Tokyo, Japan) (40), which contains chicken $\beta$-actin gene (CAG) promoter and loxP-neo-loxP. Transgenic mice (LIF-loxP mice) were generated by pronuclear microinjection. The transgene was identified by PCR analysis (forward, 5'-GAGGATCGTTTCGCATGATT-3'; reverse, 5'-TATTCGGCAAGCAGGCATCG-3'). LIF-loxP mice were crossed with MHC-Cre mice (a gift from Michael D. Schneider, Imperial College London, London, United Kingdom) (41) to generate LifuMHC-Cre mice.

Generation of sympathetic nerve-specific $g p 130$ gene-targeted mice. The $g p 130^{f l o x} f$ lox mice (42) and DBH-Cre mice (43) were provided by Werner Müller (GBF mbH, Braunschweig, Germany) and Günther Schütz (German Cancer Research Center, Heidelberg, Germany), respectively. The CAG-chloramphenicol acetyl transferase-EGFP (CAG-CAT-EGFP) transgenic mice, carrying the reporter gene construct CAG-CAT-EGFP (44), were a gift from J. Miyazaki (Osaka University, Osaka, Japan). Double-transgenic mice were generated by cross-

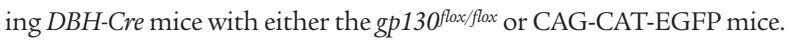

Preparation of cardiomyocyte-conditioned media. Primary culture of neonatal Wistar rat cardiomyocytes was performed as described previously (45). Cardiomyocytes were incubated in medium containing serum for 24 hours and then the medium was replaced with fresh serum-free medium. After 24 hours with or without Ang II $\left(1 \times 10^{7} \mathrm{M}\right)$, the conditioned media was collected.

Heart failure was simulated in vitro by exposing cardiomyocytes to $1 \times 10^{-7} \mathrm{M}$ Ang II (Sigma-Aldrich) for 30 minutes, $50 \mu \mathrm{mol} / 1 \mathrm{H}_{2} \mathrm{O}_{2}$ for 3 hours (Wako), $1 \mu \mathrm{mol} / 1$ doxorubicin (Sigma-Aldrich) for 2 hours, $10 \mu \mathrm{mol} / 1$ $\mathrm{NE}$ (Sigma-Aldrich) for 30 minutes, or $5 \mu \mathrm{mol} / 1$ ceramide for 15 minutes (Sigma-Aldrich). For hypoxic stimulation, the cells were cultured in a $90 \%$ $\mathrm{N}_{2} / 5 \% \mathrm{CO}_{2} / 5 \% \mathrm{O}_{2}$ atmosphere for 2 hours. For mechanical stretch stimu- 
A

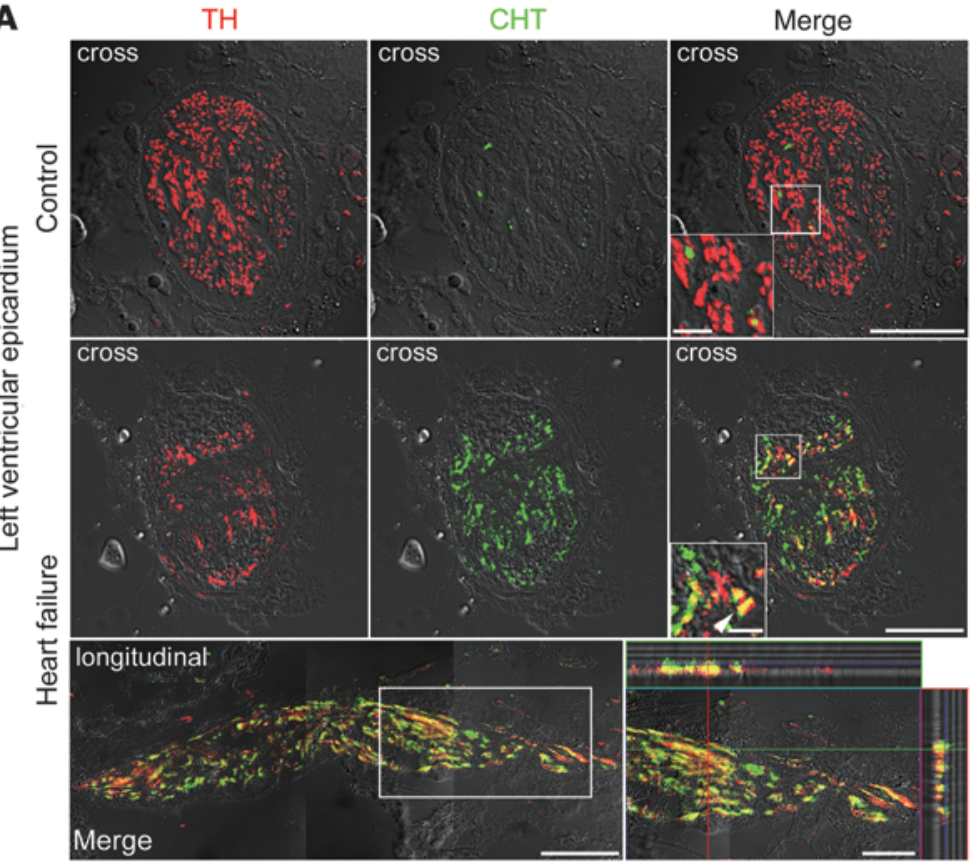

C

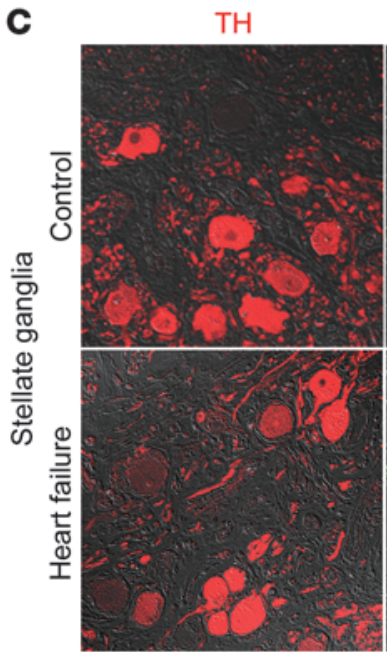

ChAT

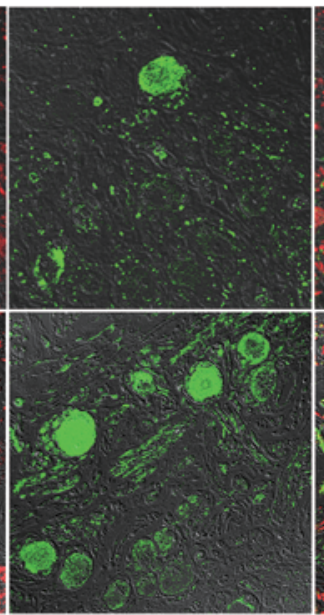

Merge

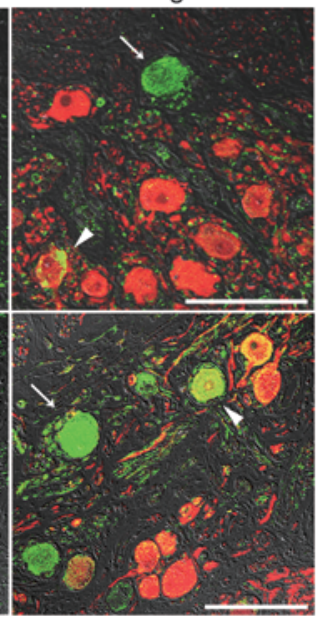

B
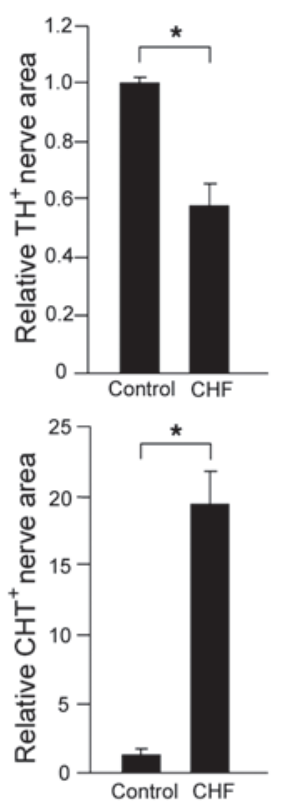

D

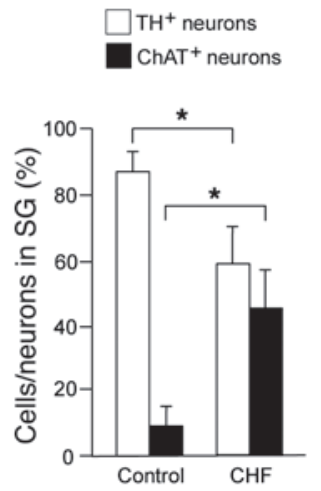

Figure 9

Neurotransmitter switching in the human cardiac sympathetic nervous system. (A) Representative immunostaining for TH (red) and CHT (green) in LVs at the epicardial sites in control and heart failure patients. Cross and longitudinal sections are shown. The longitudinal image is composed of 3 serial images. The heart failure group has fewer $\mathrm{TH}^{+}$nerves and markedly more $\mathrm{CHT}^{+}$nerves than the control group. In the ventricle of heart failure patients, some $\mathrm{CHT}^{+}$nerves coexpress $\mathrm{TH}$ (arrowhead). The longitudinal section also revealed sympathetic nerves coexpressing $\mathrm{TH}$ and $\mathrm{CHT}$. Higher-magnification views of the boxed regions are shown in the insets (right panels). (B) Quantitative analysis of $\mathrm{TH}^{+}$and $\mathrm{CHT}^{+}$nerve areas in LV $(n=4)$. (C) Representative immunostaining for TH (red) and ChAT (green) in the stellate ganglia of control and heart failure patients. Arrows indicate ChAT ${ }^{+}$cells, and arrowheads indicate $\mathrm{TH}^{+} / \mathrm{ChAT}^{+}$neurons. The heart failure group has fewer $\mathrm{TH}^{+}$cells and more $\mathrm{ChAT}^{+}$cells than the control group. (D) Quantification of $\mathrm{TH}^{+}$and $\mathrm{ChAT}^{+}$cells per total number of neurons in the stellate ganglia $(n=5)$. Representative data are shown in each panel. ${ }^{*} P<0.01$. Scale bars: $10 \mu \mathrm{m}$ (A, insets); $50 \mu \mathrm{m}$ (A and inset in $\left.\mathbf{D}\right) ; 100 \mu \mathrm{m}$ (C).

lation, cardiomyocytes were cultured on laminin-coated silicon dishes that were stretched by $20 \%$ for 1 hour.

Lif and Ctf1 siRNA transfection. Lif and Ctf1 siRNA and negative control (scrambled) siRNA were purchased from Ambion. Cardiomyocytes were transfected using the Lipofectamine RNAiMAX reagent (Invitrogen).

Culture of sympathetic neurons. Stellate ganglia neurons from 2-dayold Wistar rats (Japan CLEA) were dissociated and cultured in DMEM (Sigma-Aldrich) containing 5\% fetal bovine serum and nerve growth factor
(50 ng/ml; Upstate), as described previously $(8,9)$. Proliferation of nonneuronal cells was prevented by addition of $10 \mu \mathrm{M}$ cytosine $1 \beta$-D-arabino furanoside (Sigma-Aldrich) for the first 5 days. After 2 days, the neurons were cultured with LIF (Chemicon) or cardiomyocyte-conditioned media for 14 days. The culture media was replaced every 2-3 days.

$R N A$ extraction and quantitative RT-PCR. RNA extraction and quantitative RT-PCR were performed as described previously (45) using the ABI Prism 7500 Sequence Detection System (Applied Biosystems). All samples were 
run in triplicate. The primers and TaqMan probe for LIF (Rn00573491_ g1, Mm00434762_g1), CT-1 (Rn00567503_m1, Mm00432772_m1), ciliary neurotrophic factor (Rn00755092_m1), cardiotrophin-like cytokine (Rn02133709_s1), nerve growth factor (Rn02133709_s1), brain-derived neurotrophic factor (Rn01484928_m1), neurotrophin-3 (Rn00579280_ m1), GDNF (Rn00569510_m1), VAChT (Rn00581454_s1), CHT (Rn00506029_m1), TH (Rn00562500_m1), brain natriuretic peptide (Rn00580641_m1, Mm00435304_g1), angiotensinogen (Rn00593114_ $\mathrm{m} 1$ ), and ACE (Rn00561094_m1) were purchased from Applied Biosystems. The mRNA levels were normalized by comparison to GAPDH.

RT-PCR. The primers for ChAT (46) and VAChT (47) were as described previously. The primers for murine LIF were murine LIF forward, 5'-CCTCTAGAGTCCAGCCCATAA- $3^{\prime}$, and murine LIF reverse, $5^{\prime}$-CTCTAGAAGGCCTGGACCAC-3'.

Western blot analysis. Samples were prepared by homogenization of hearts in ice-cold buffer as described (45). Immunodetection was performed with an anti-LIF antibody (AB-449-NA; R\&D Systems). After transfer to nitrocellulose membranes, LIF protein was visualized by chemiluminescence detection (SuperSignal West Pico; Pierce).

Immunohistochemistry. Hearts were perfused from the apex with $0.4 \%$ paraformaldehyde in PBS, fixed overnight, embedded in OCT compound, and then frozen in liquid nitrogen. Cryostat sections were stained with antibodies against TH (Chemicon, Sigma-Aldrich, and ImmunoStar) and DBH to detect sympathetic nerves, with CHT (Chemicon) and ChAT (Chemicon) to detect parasympathetic nerves, and with calcitonin gene-related peptide (Biogenesis) to detect sensory nerves. The sections were incubated with secondary antibodies conjugated with Alexa Fluor 488, Alexa Fluor 546, Alexa Fluor 633 (Molecular Probes) and TRITC (DAKO), and the nuclei were stained with Toto3 (Molecular Probes). All confocal microscopy was carried out on a LSM 510 META Confocal Microscope (Carl Zeiss). In some experiments, paraffin-embedded sections were treated with $10 \mathrm{mM}$ citrate buffer ( $\mathrm{pH}$ 6) and $20 \mathrm{mM}$ Tris-HCL buffer ( $\mathrm{pH}$ 9) for antigen retrieval, and signals were visualized using a TSA Direct Kit (NEN Life Science). Nerve density was determined using ImageJ software (http://rsbweb.nih.gov/ij/) (48). For human sympathetic ganglia, cresyl violet staining (Nissl staining) was performed, and the total number of neurons was determined.

Anterograde labeling of sympathetic nerve fibers. To label sympathetic nerve fibers in the stellate ganglia, DS and DR rats were injected with $10 \%$ BDA in the left stellate ganglia, via a Hamilton micropipette under mechanical ventilation. The rats were killed after 3 days and were perfused with $0.4 \%$ paraformaldehyde in PBS for immunohistochemistry.

Transmission electron microscopy. Hearts were fixed with $0.2 \%$ glutaraldehyde and $0.4 \%$ paraformaldehyde in $0.1 \mathrm{M}$ cacodylate buffer and embedded in epoxy resin. Ultrathin sections $(80 \mathrm{~nm})$ were stained as described previously (49) and viewed under a JEOL-1230 transmission electron microscope (JEOL Ltd.). AF64A was prepared from acetylethylcholine mustard hydrochloride (Sigma-Aldrich) and administered to rats intraperitoneally $(150 \mu \mathrm{mol} / \mathrm{kg})$. AF64A is specifically taken up by CHT and degenerates cholinergic nerve fibers. Saline was injected in control experiments. After 3 days, hearts were removed and fixed as above. The samples were immunostained with an antibody against TH (Chemicon) and incubated with a secondary antibody of Nanogold-Fab conjugates (Nanoprobes). The conjugated gold particles were visualized as described previously (50).
Mouse models of heart failure. LV failure was induced by TAC, which was performed by ligating the transverse thoracic aorta with a 27 -gauge needle, using a 7.0 nylon suture (51). RV failure was induced by long-standing hypoxia $\left(10 \% \mathrm{O}_{2}\right)$ in the mouse model of pulmonary hypertension as described previously (52).

Echocardiography and hemodynamic measurements. Transthoracic echocardiography was performed with a Vevo 770 scanner (VisualSonics), using 17.5 and $30 \mathrm{MHz}$ probes. TAC pressure gradients were determined by pulsed-wave Doppler echocardiography and calculated as $4 \times V_{\max }{ }^{2}$, where $V_{\max }$ is the velocity of the blood across the constriction. Mice with a pressure gradient of more than $40 \mathrm{mmHg}$ were included in the study. For hemodynamic measurements, the mice were intubated and then mechanically ventilated with a respirator. A left thoracotomy was performed, and $\mathrm{RV}$ pressure was measured from a 1.0F Mikro-Tip pressure transducer (SPR-1000; Millar Instruments), directly inserted into the RV. The LV and aortic pressures were measured by catheters inserted into the right carotid artery. $\mathrm{LV}$ pressure, $\mathrm{RV}$ pressure, and aortic pressure were recorded using a polygraph system (iWorx Systems Inc.). All recordings were performed on rats and mice anesthetized with $2 \%$ isoflurane.

Human samples. Control samples of LV and stellate ganglia were prepared from 8 autopsied patients without heart disease (mean age, $57 \pm 17$ years). Heart failure samples were prepared from 8 autopsied patients (mean age, $51 \pm 13$ years), who died of CHF due to various heart diseases, as described in Table 1. After removal samples were fixed immediately in $0.4 \%$ paraformaldehyde and embedded in paraffin or OCT compound. The use of autopsied specimens of human tissue was approved by the institutional review board of Keio University and National Cardiovascular Center.

Statistics. Values are presented as mean \pm SD. Statistical significance was evaluated using 2-tailed, unpaired Student's $t$ tests for comparisons of 2 mean values. $P$ values of less than 0.05 were considered statistically significant. Kaplan-Meier survival curves were analyzed by SPSS software. Survival analysis was performed by using the Kaplan-Meier method. The log-rank test was used to compare survival curves.

\section{Acknowledgments}

We thank Yoshiko Miyake, Yoko Shiozawa, Seiichi Kotoda, Yoshiharu Tsuru, Toshihiro Nagai, Koji Shimoda, and Toshio Terashima for excellent technical assistance. We are also grateful to Kazuto Yamazaki, Keiko Fukada, and Takeshi Suzuki for their comments on the manuscript. This study was supported in part by research grants from the Ministry of Education, Science and Culture, Japan, and by the Program for Promotion of Fundamental Studies in Health Science of the National Institute of Biomedical Innovation (to K. Fukuda).

Received for publication May 6, 2009, and accepted in revised form November 11, 2009.

Address correspondence to: Keiichi Fukuda, Department of Regenerative Medicine and Advanced Cardiac Therapeutics, Keio University School of Medicine, 35 Shinanomachi, Shinjuku-ku, Tokyo 160-8582, Japan. Phone: 81-3-5363-3874; Fax: 81-3-53633875; E-mail:kfukuda@sc.itc.keio.ac.jp.
1. Cao JM, et al. Relationship between regional car-
diac hyperinnervation and ventricular arrhythmia.
Circulation. 2000;101(16):1960-1969.
2. Bristow MR, et al. Beta-adrenergic neuroeffector
abnormalities in the failing human heart are pro-
duced by local rather than systemic mechanisms.
JClin Invest. 1992;89(3):803-815.
3. Chidsey CA, Kaiser GA, Sonnenblick EH, Spann JF,
Braunwald E. Cardiac norephinephrine stores in experimental heart failure in the dog. J Clin Invest. 1964;43:2386-2393.

4. Backs J, et al. The neuronal norepinephrine transporter in experimental heart failure: evidence for a posttranscriptional downregulation. J Mol Cell Cardiol. 2001;33(3):461-472.

5 . Himura Y, et al. Cardiac noradrenergic nerve termi- nal abnormalities in dogs with experimental congestive heart failure. Circulation. 1993;88(3):1299-1309.

6. Kimura K, et al. Cardiac Sympathetic Rejuvenation: A Link between Nerve Function and Cardiac Hypertrophy. Circ Res. 2007;100(12):1755-1764.

7. Francis NJ, Landis SC. Cellular and molecular determinants of sympathetic neuron development. Annu Rev Neurosci. 1999;22:541-566. 
8. Patterson PH, Chun LL. The influence of nonneuronal cells on catecholamine and acetylcholine synthesis and accumulation in cultures of dissociated sympathetic neurons. Proc Natl Acad Sci U S A. 1974;71(9):3607-3610

9. Fukada K. Hormonal control of neurotransmitter choice in sympathetic neurone cultures. Nature. 1980;287(5782):553-555.

10. Fukada K. Purification and partial characterization of a cholinergic neuronal differentiation factor. Proc Natl Acad Sci U S A. 1985;82(24):8795-8799.

11. Yamamori $\mathrm{T}$, et al. The cholinergic neuronal differentiation factor from heart cells is identical to leukemia inhibitory factor. Science. 1989; 246(4936):1412-1416.

12. Stanke M, et al. Target-dependent specification of the neurotransmitter phenotype: cholinergic differentiation of sympathetic neurons is mediated in vivo by gp 130 signaling. Development. 2006; 133(1):141-150.

13. Bamber BA, Masters BA, Hoyle GW, Brinster RL, Palmiter RD. Leukemia inhibitory factor induces neurotransmitter switching in transgenic mice. Proc Natl Acad Sci U S A. 1994;91(17):7839-7843.

14. Blum A, Miller H. Role of cytokines in heart failure. Am Heart J. 1998;135(2 Pt 1):181-186.

15. Hirota $\mathrm{H}$, et al. Circulating interleukin- 6 family cytokines and their receptors in patients with congestive heart failure. Heart Vessels. 2004;19(5):237-241.

16. MacGowan GA, Mann DL, Kormos RL, Feldman AM, Murali S. Circulating interleukin-6 in severe heart failure. Am J Cardiol. 1997;79(8):1128-1131.

17. Ieda $M$, et al. Sema3a maintains normal heart rhythm through sympathetic innervation patterning. Nat Med. 2007;13(5):604-612.

18. Potter DD, Landis SC, Matsumoto SG, Furshpan EJ. Synaptic functions in rat sympathetic neurons in microcultures. II. Adrenergic/cholinergic dual status and plasticity. J Neurosci. 1986;6(4):1080-1098.

19. Fisher A, Mantione CR, Abraham DJ, Hanin I. Longterm central cholinergic hypofunction induced in mice by ethylcholine aziridinium ion (AF64A) in vivo. J Pharmacol Exp Ther. 1982;222(1):140-145.

20. Zigmond RE, et al. Phenotypic plasticity in adult sympathetic neurons: changes in neuropeptide expression in organ culture. Proc Natl Acad SciU S A. 1992;89(4):1507-1511.

21. Furshpan EJ, Landis SC, Matsumoto SG, Potter DD. Synaptic functions in rat sympathetic neurons in microcultures. I. Secretion of norepinephrine and acetylcholine. J Neurosci. 1986;6(4):1061-1079.

22. Delree $P$, et al. Plasticity of developing and adult dorsal root ganglion neurons as revealed in vitro. Brain Res Bull. 1993;30(3-4):231-237.

23. Hasking GJ, et al. Norepinephrine spillover to plasma in patients with congestive heart failure: evidence of increased overall and cardiorenal sympathetic nervous activity. Circulation.
1986;73(4):615-621.

24. Meredith IT, et al. Cardiac sympathetic nervous activity in congestive heart failure. Evidence for increased neuronal norepinephrine release and preserved neuronal uptake. Circulation. 1993 88(1):136-145

25. Merlet $\mathrm{P}$, et al. Prognostic value of cardiac metaiodobenzylguanidine imaging in patients with heart failure. J Nucl Med. 1992;33(4):471-477.

26. Henderson EB, et al. Abnormal I-123 metaiodobenzylguanidine myocardial washout and distribution may reflect myocardial adrenergic derangement in patients with congestive cardiomyopathy. Circulation. 1988;78(5 Pt 1):1192-1199.

27. Qin F, Vulapalli RS, Stevens SY, Liang CS. Loss of cardiac sympathetic neurotransmitters in heart failure and NE infusion is associated with reduced NGF. Am J Physiol Heart Circ Physiol. 2002; 282(1):H363-H371.

28. Kaye DM, Vaddadi G, Gruskin SL, Du XJ, Esler MD Reduced myocardial nerve growth factor expression in human and experimental heart failure. Circ Res. 2000;86(7):E80-E84.

29. Schmid P G, et al. Selective sympathetic neural changes in hypertrophied right ventricle. Am J Physiol. 1982;243(2):H175-H180.

30. Liang CS, Yatani A, Himura Y, Kashiki M, Stevens SY. Desipramine attenuates loss of cardiac sympathetic neurotransmitters produced by congestive heart failure and NE infusion. Am J Physiol Heart Circ Physiol. 2003;284(5):H1729-H736.

31. Wang H, Lu Y, Wang Z. Function of cardiac M3 receptors. Auton Autacoid Pharmacol. 2007;27(1):1-11.

32. LaCroix C, Freeling J, Giles A, Wess J, Li YF. Deficiency of M2 muscarinic acetylcholine receptors increases susceptibility of ventricular function to chronic adrenergic stress. Am J Physiol Heart Circ Physiol. 2008;294(2):H810-H820.

33. Li M, et al. Vagal nerve stimulation markedly improves long-term survival after chronic heart failure in rats. Circulation. 2004;109(1):120-124.

34. Rao MS, et al. Leukemia inhibitory factor mediates an injury response but not a target-directed developmental transmitter switch in sympathetic neurons. Neuron. 1993;11(6):1175-1185.

35 . Berry MF, et al. Targeted overexpression of leukemia inhibitory factor to preserve myocardium in a rat model of postinfarction heart failure. J Thorac Cardiovasc Surg. 2004;128(6):866-875.

36. Brodski C, Schnurch H, Dechant G. Neurotrophin-3 promotes the cholinergic differentiation of sympathetic neurons. Proc Natl Acad Sci U S A. 2000;97(17):9683-9688.

37. Brodski C, Schaubmar A, Dechant G. Opposing functions of GDNF and NGF in the development of cholinergic and noradrenergic sympathetic neurons. Mol Cell Neurosci. 2002;19(4):528-538.

38. Jougasaki M, et al. Leukemia inhibitory factor is augmented in the heart in experimental heart failure. Eur J Heart Fail. 2003;5(2):137-145.

39. Eiken HG, et al. Myocardial gene expression of leukaemia inhibitory factor, interleukin-6 and glycoprotein 130 in end-stage human heart failure. Eur J Clin Invest. 2001;31(5):389-397.

40. Kanegae Y, et al. Efficient gene activation system on mammalian cell chromosomes using recombinant adenovirus producing Cre recombinase. Gene. 1996;181(1-2):207-212.

41. Agah R, et al. Gene recombination in postmitotic cells: targeted expression of Cre recombinase provokes cardiac-restricted, site-specific rearrangement in adult ventricular muscle in vivo. J Clin Invest. 1997;100(1):169-179.

42. Betz UA, et al. Postnatally induced inactivation of gp130 in mice results in neurological, cardiac, hematopoietic, immunological, hepatic, and pulmonary defects. J Exp Med. 1998;188(10):1955-1965.

43. Parlato R, Otto C, Begus Y, Stotz S, Schütz G. Specific ablation of the transcription factor CREB in sympathetic neurons surprisingly protects against developmentally regulated apoptosis. Development. 2007;134(9):1663-1670

44. Kawamoto S, et al. A novel reporter mouse strain that expresses enhanced green fluorescent protein upon Cre-mediated recombination. FEBS Lett. 2000;470(3):263-268.

45. Ieda M, et al. Endothelin-1 regulates cardiac sympathetic innervation in the rodent heart by controlling nerve growth factor expression. J Clin Invest. 2004;113(6):876-884.

46. Fann MJ, Patterson PH. A novel approach to screen for cytokine effects on neuronal gene expression. J Neurochem. 1993;61(4):1349-1355.

47. Berrard S, et al. Coregulation of two embedded gene products, choline acetyltransferase and the vesicular acetylcholine transporter. J Neurochem. 1995; 65(2):939-942.

48. Ieda $M$, et al. Nerve growth factor is critical for cardiac sensory innervation and rescues neuropathy in diabetic hearts. Circulation. 2006;114(22):2351-2363.

49. Yamazaki K, Eyden BP. Gap junctions and nerve terminals among stromal cells in human fallopian tube ampullary mucosa. J Submicrosc Cytol Pathol. 1998;30(3):399-408.

50. Nakata K, Okuda T, Misawa H. Ultrastructural localization of high-affinity choline transporter in the rat neuromuscular junction: enrichment on synaptic vesicles. Synapse. 2004;53(1):53-56.

51. Rockman HA, et al. Segregation of atrial-specific and inducible expression of an atrial natriuretic factor transgene in an in vivo murine model of cardiac hypertrophy. Proc Natl Acad Sci U S A. 1991; 88(18):8277-8281.

52. Endo J, et al. BM-derived cells contribute to the pathogenesis of cardiac hypertrophy in response to pressure overload. Circulation. 2007;116(10):1176-1184. 